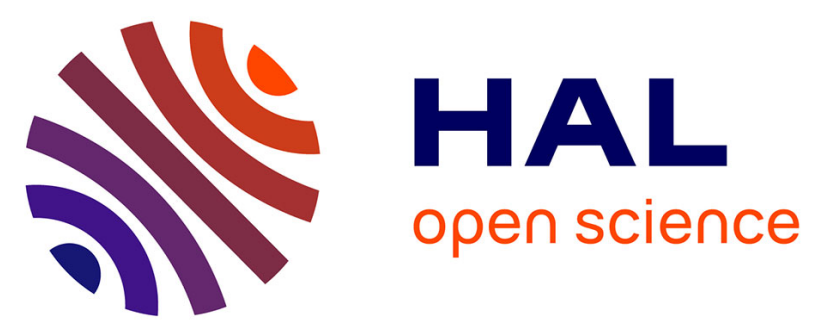

\title{
The Size Advantage Model of Sex Allocation in the Protandrous Sex-Changer Crepidula fornicata: Role of the Mating System, Sperm Storage, and Male Mobility
}

Thomas Broquet, Audrey Barranger, Emmanuelle Billard, Anastasia Bestin, Rémy Berger, Gaelle Honnaert, Frédérique Viard

\section{To cite this version:}

Thomas Broquet, Audrey Barranger, Emmanuelle Billard, Anastasia Bestin, Rémy Berger, et al.. The Size Advantage Model of Sex Allocation in the Protandrous Sex-Changer Crepidula fornicata: Role of the Mating System, Sperm Storage, and Male Mobility. The American Naturalist, 2015, 186 (3), pp.404-420. 10.1086/682361 . hal-01196801

\section{HAL Id: hal-01196801 https: / hal.sorbonne-universite.fr/hal-01196801}

Submitted on 10 Sep 2015

HAL is a multi-disciplinary open access archive for the deposit and dissemination of scientific research documents, whether they are published or not. The documents may come from teaching and research institutions in France or abroad, or from public or private research centers.
L'archive ouverte pluridisciplinaire HAL, est destinée au dépôt et à la diffusion de documents scientifiques de niveau recherche, publiés ou non, émanant des établissements d'enseignement et de recherche français ou étrangers, des laboratoires publics ou privés. 
1 The size advantage model of sex allocation in the protandrous sex-changer Crepidula fornicata: role of the mating system, sperm storage, and male mobility

4 Honnaert, Frédérique Viard.

5 CNRS, team diversity and connectivity of coastal marine landscapes, Station Biologique de Roscoff, 629680 Roscoff, France.

7 and

8 Sorbonne Universités, UPMC Univ Paris 06, UMR 7144, Station Biologique de Roscoff, 29680 Roscoff, 9 France

E-mail addresses: thomas.broquet@sb-roscoff.fr; audrey.barranger@gmail.com;

e.billard@gmail.com; abestin@rennes.inra.fr; remy.berger@laposte.net;

gaelle_honnaert@hotmail.com; viard@sb-roscoff.fr S7, supplementary references, and color version of figure 1. 
23 Sequential hermaphroditism is adaptive when the reproductive value of individuals varies with size

24 or age and this relationship differs between males and females. In this case theory shows that the

25 lifetime reproductive output of an individual is increased by changing sex (a hypothesis referred to

26 as the size-advantage model). Sex-linked differences in size-fitness curves can stem from differential

27 costs of reproduction, the mating system, and differences in growth and mortality between sexes.

28 Detailed empirical data is required to disentangle the relative roles of each these factors within the theory. Quantitative data are also needed to explore the role of sperm storage, which has not yet been considered with sequential hermaphrodites. Using experimental rearing and paternity assignment, we report relationships between size and reproductive success of Crepidula fornicata, a protandrous (male-first) gastropod. Male reproductive success increased with size due to the polygamous system and stacking behavior of the species, but females nonetheless had greater reproductive success than males of the same size, in agreement with the size-advantage theory. Sperm storage appeared to be a critical determinant of success for both sexes, and modeling the effect of sperm storage showed that it could potentially accelerate sex-change in protandrous species. 

point during their life. This strategy is adaptive when the lifetime reproductive output of an individual is maximized by changing sex during its life. Theory shows that this happens when the gender that provides the highest reproductive value (expected future reproduction) differs depending on the size or the age of the individual (the size advantage hypothesis, Ghiselin 1969; Warner 1988). This hypothesis has been formalized under the general framework of sex allocation theory (reviewed in Charnov 1982; West 2009), which correctly predicts both the direction and timing of sex-change in sequential hermaphrodites from several groups where this reproductive strategy is found.

The size advantage model has broad applicability and is seemingly simple since it can be outlined by the relationship between reproductive value and a unique predictor (body size or age). However, it is important to realize that this relationship integrates a range of complex biological processes that drive reproduction expectations of individuals of both sexes (discussed e.g. in Charnov 1982; Warner 1988; West 2009). The reproductive value of an individual has several components: it integrates both current and future reproductive output, and the probability of surviving to reproduce in the future. There are thus several mechanisms that can result in males and females displaying different reproductive value trajectories with respect to age/size. of these gametes requires space within the body (e.g. in fish and invertebrates). In addition the female function often entails higher energy costs than the male function. Female fecundity therefore increases sharply with body size in many species. If this size-effect results in large individuals having a higher reproductive value as females than as males, then protandry (male-first sex change) is selected for. 

reproductive value being greater in one sex, thereby introducing selection for sex change. Theory shows that there is an advantage to starting life as the sex that has the fastest growth rate and/or the lowest mortality rate (e.g. Charnov 1982, p. 136; Iwasa 1991).

Finally, social systems can create conditions where a differential effect of size on male vs female reproductive success can be expressed (Munday et al. 2006). In this study we focused on this factor, which is perhaps more elusive than the first two factors because species that change sex have a variety of mating systems and sexual selection regimes. A general finding is that polygynous mating systems favor protogynous (female-first) sex change, because larger individuals have a significant advantage in male-male competition (Ghiselin 1969; Andersson 1994; Munday et al. 2006; West 2009). Specifically, protogyny is selected for when the advantage of large males is strong enough to result in an individual's reproductive success increasing more rapidly with size for males than for females. On the other hand, protandry (male-first sex change) is adaptive if the increase in male reproductive success as a function of size does not outweigh the fecundity advantage of large females. In line with this, mating systems where size has little or no effect on male reproductive success are associated with protandrous species (e.g. Charnov 1982, p.142, 182; West 2009, p.200, 223).

Empirical research has provided several illuminating examples that have validated the sizeadvantage theory and the expected effects of different mating systems within this theory (e.g. protandrous pandalid shrimps and protogynous labroid fish, reviewed and analyzed in details in Charnov 1982). However, other social systems remain to be examined and authors have repeatedly called for more quantitative data on sex-specific size-fitness functions (e.g. West 2009; Kazancioglu and Alonzo 2010). the effect of social systems on sex change relative to other factors. Slipper limpets are bentho- 
pelagic mollusks (i.e. sedentary adults producing free-living larvae). They form long-lived stacks of typically 2 to 20 individuals with younger, smaller individuals (immature or males) piling up on older, larger ones (females). These stacks are permanent mating associations of genetically unrelated individuals of various ages and sexes that reproduce with each other (individuals may live up to ca. 10 years). All individuals mature first as males before changing sex at a time that depends strongly on environmental (social) conditions (earliest work by Conklin 1897; Orton 1912; Coe 1936; Hoagland 1978). Interestingly, parentage studies (starting with Gaffney and McGee 1992) have repeatedly shown that the mating system is polygamous and male reproductive success may be highly variable (suggested by results from Dupont et al. 2006; Proestou et al. 2008; Le Cam et al. 2009). This variance seems to be linked with the position of individuals within stacks: the stacking process results in older larger males being closer to the females and having higher reproductive success (Dupont et al. 2006; Proestou et al. 2008; Le Cam et al. 2009). The oldest / largest males are thus possibly at a strong advantage, as suggested by Dupont et al. (2006) and Proestou et al. (2008) but is not clear how this tendency in males compares with the strong size-fitness relationship reported for females (Hoagland 1978; Richard et al. 2006; Li and Pechenik 2007; Proestou et al. 2008; Le Cam and Viard 2011). It is thus interesting to quantify the reproductive success of males and females of different sizes and test whether the suspected increase of male reproductive success with size is verified: a strong size-effect on male reproductive success could be compatible with protandry if large females have even greater reproductive success than large males.

Moreover, reproductive success is only part of the story. The reproductive value of males and females also depends on other factors that could be of importance (e.g. growth and mortality). In particular, male slipper limpets grow faster than females of the same size (Collin 1995), a situation that favors protandrous sex-change. Mollusks (and particularly Calyptraeidae, the family that contains Crepidula species) have been the focus of much effort to disentangle the selective forces driving sex-change (Collin 2013). Yet quantitative data on fitness have remained difficult to obtain and the lack thereof is a persistent barrier to understanding the role of mating systems in sex 
allocation. In Crepidula fornicata as in most other species, such investigations have been limited so far by the relatively low number of broods and adults used in parentage analyses and, consequently, the lack of precise quantitative estimates for male and female reproductive success.

One additional mating-system-linked factor that might be of relevance to both the direction and the timing of sex change in Crepidula and many other sex-changing species is sperm storage. Female Crepidula fornicata can mate repeatedly and produce several broods over a reproductive season using sperm from multiple males that has been stored, possibly over a long time period (at least one year according to Hoagland 1978, see also discussion in Conklin 1897). Although sperm storage does affect the variance in reproductive success of individuals of both sexes (e.g. Dupont et al. 2006), its effect on the evolution of sequential hermaphroditism has been largely ignored so far, not only in limpets (see, however, discussions in Dupont et al. 2006; Proestou et al. 2008) but to our knowledge also in any other organism. This is in strong contrast with the situation of simultaneous hermaphroditism, where sperm storage was shown to play an important role (Charnov 1996; Michiels 1998; Angeloni et al. 2002). Variations in sperm production, sperm-holding capacity, and investigation.

Finally, there is an interesting additional twist to the sex change system in Crepidula fornicata. There have been recurrent discussions in the literature regarding whether the mobility of a male 1912; Coe 1938). This led several authors to wonder whether small mobile could have a substantially 
argued against (Collin 1995) and is either not supported or only poorly supported by genetic parentage studies (Dupont et al. 2006; Proestou et al. 2008; Le Cam et al. 2009). Yet these parentage studies have repeatedly found that a small fraction of larvae were sired by males that were not present in the maternal stack. The role of such small males, which may fertilize females by wandering on the sides of other individuals and roving from stack to stack, remains controversial.

The primary goal of this study was to assess the role of the mating system in determining sizereproductive success relationships in a protandrous sex changer in the context of the size advantage theory. To attain this objective, we sampled Crepidula fornicata stacks in the wild and maintained them in the lab in an experimental set-up that allowed us to: 1) sample larvae as they were released (several successive broods per female), 2) estimate the reproductive success of males and females through genetic parentage analyses, and 3) identify the determinants of reproductive success with regard to the size advantage model of sex-change. Because sperm storage appeared to have a very important influence on the reproductive success of the study species, we developed a model to test whether sperm storage could theoretically have a significant effect on the timing of sex-change in 153 protandrous hermaphrodites. Finally, observations of an unexpected behavior for several small mobile males prompted us to investigate the effect of male mobility on reproductive success and sex-change. 
Adult Crepidula fornicata were sampled in the wild within an area of a few hundred square meters (Baie de Morlaix, North-west Brittany, France) on January 12, 2011. This period corresponds to the very beginning of the reproductive season for C. fornicata in this area (Richard et al. 2006; Le Cam 2009, p. 132-133). Stacks were sampled one-by-one by scuba-divers at a depth of $14 \mathrm{~m}$ within a period of one hour. Two sets of stacks were used in this study. First, 33 stacks of 4 to 15 individuals were selected for our rearing experiment (294 individuals overall). Second, 21 additional stacks of 4 to 15 ind. (total: 190 ind.) were fixed in ethanol and later used to estimate allelic frequencies in the source population. Data from the additional stacks provided an independent reference for downstream parentage analyses (see below and supplementary material). (conditions detailed in supplementary material). Each stack was placed in an individual plastic structure sealed near the bottom with a net (200 $\mu \mathrm{m}$ mesh size) through which water could freely circulate while all released larvae would be retained (Fig.1). Each event of larval release could thus easily be detected and associated with one particular parental stack. of 33 stacks. The eight remaining stacks were chosen for in-depth analyses of reproductive success (Table 1) and for logistic reasons they were removed earlier (after 85 days) or later (286 days). Many occurrences of mating were randomly observed over the course of the experiment. Mating partners were identified, and the distance between mating individuals was defined as the number of other limpets (male or female) separating them (i.e. two individuals in direct contact would be at a distance of 0). All larval releases were recorded (see below). Finally, the death of individuals was 
recorded (especially during cleaning operations, when most dead individuals would detach from the

182

183 stack).

Adult traits and genotyping

All adults were fixed in ethanol either when they were found dead during the course of the experiment (see Results), or at the end of the experiment. Each individual was then sexed according to Hoagland (1978), its position in a stack was recorded, the presence of brooded egg sacs was noted, and a tissue sample was taken for genetic analyses. All adults (along with the additional 190 individuals that had been simultaneously sampled in the wild) were genotyped at 9 microsatellite loci: DAYN22, BI13YE17, DA8YN14, CL322, DA5YM24, CL191, CL270, DA4Y003 (Riquet et al. 2011), and $\mathrm{CfH} 7$ (Dupont et al. 2006) following the protocols detailed by the authors.

The size of each individual, defined as the shell straight length $L$ (largest antero-posterior shell length, following e.g. Richard et al. 2006) was measured with a caliper on the first day of the experiment and after the death of the individual. Shell growth was estimated as the difference between these two measurements. We used the individuals that were reared for 251 days (and alive at this date) to estimate shell growth as a function of the initial size of individuals and their sex.

These data were further used to approximate Von Bertalanffy growth parameters without distinguishing between sexes in order to obtain an average growth model that was used in our modeling study (see details in the model section).

\section{Sampling of larvae}

Swimming larvae were visible immediately after their release, but because they were contained within the aquariums with the adults they tended to be filtered by the adults (as observed by Pechenik et al. 2004), leading to their being lost over about a 24-hour period. As our goal was to estimate the reproductive success of individuals, we surveyed the presence of larvae as often as 
possible: several times per day and at least once every night (around 23h) during the week, and at least once during week-ends.

The eight stacks chosen for in-depth analyses of reproductive success (Table 1) were surveyed intensively until the desired number of larval releases was observed. Note that a single release of larvae could have been a mixture of two broods if two females had released their larvae simultaneously. Similarly, two successive batches of larvae released over a short interval (a few hours) could have corresponded to a single brood. To avoid confusion, we will use the term "larval release" to designate an observation of a batch of larvae in the experimental setup, while the word "brood" will designate the actual brood of one given female (which could only be fully defined after parentage analyses). At this stage of the experiment we did not know the sex of the individuals within the stacks (live individuals cannot be sexed). Based on the number of individuals per stack and prior knowledge of sex-ratio distribution in the sampled population, we aimed for ca. 8 broods per stack in order to obtain more than one brood per female and thus estimate reproductive success of most males and females. To this end, we obtained data from the first 6 to 9 larval releases of each stack (62 in total, see Table 1 and Results).

Each observation of a larval release was treated as follows: 1) all larvae were immediately collected in a measuring cylinder filled with seawater (typical volume $V=500 \mathrm{~mL}$ ). 2) After gentle mixing, the larvae contained in several (at least five) $3 \mathrm{~mL}$ subsamples were exhaustively counted under a binocular microscope. An estimation of the number of larvae released is then $N=\bar{x} \times V$, with $\bar{x}$ the mean concentration of larvae across $n$ samples. The standard error of $N$ was estimated as $s_{N}=\frac{s_{x}}{\sqrt{n-1}} \times V$, where $s_{x}$ is the standard error of larval concentrations estimated across the $n 3 \mathrm{~mL}$ samples where larvae were counted. 3) Finally, a sample of approximately 100-300 larvae was fixed in $100 \%$ ethanol for downstream genetic analyses. 

fraction could be genotyped for parentage assignment and reproductive success analyses. The probability of detecting at least one offspring of a father that contributed a fraction $p$ of a brood can be calculated as $1-(1-p)^{n}$ with sample size $n$. We therefore genotyped 48 larvae from each of the 62 larval releases mentioned above (in a few cases more larvae were genotyped). With this sample size the probability of detecting a father that sired e.g. $5 \%$ of a brood is $91.5 \%$ ( $99.3 \%$ for a father that contributed $10 \%$ of a brood, see supplementary material figure S1-A) and the number of larvae from this father expected to be present in one sample is 3 ( 5 for a father that contributed $10 \%$ of a brood, Fig. S1-B). This assumes that one larval release corresponds to one female's brood and each larva can be genetically assigned to its parents. The former assumption was not strictly fulfilled

Parentage analyses

Parentage analyses were facilitated by the fact that a small set of candidate parents (that is, all adults of a given stack) was clearly identified for each genotyped larva. However, previous parentage studies in Crepidula fornicata have repeatedly found that some larvae were fathered by males that were not present in the stack where the incubating mother was sampled (Gaffney and McGee 1992; Dupont et al. 2006; Proestou et al. 2008; Le Cam et al. 2009). Hence we used the method implemented in the software ColonY V2.0.4.5 (Jones and Wang 2010) where parentoffspring and sibship relations are simultaneously inferred (including siblings with no candidate parents in the sample pool). Each larval release (represented by 48 larvae) was analyzed independently. The parentage analysis methodology is detailed in the supplementary material. 

of larvae) and the male function (fertilization of eggs). Paradoxically, these two functions can occur simultaneously in sequential hermaphrodites. This happens when the sperm produced by a male has been stored and is being used by one or several females at a time when the male has itself switched to the female sex (note that selfing is not possible in C. fornicata: individuals do not store their own sperm after changing sex). The reproductive success of an individual slipper limpet at a given point in time has thus two components: the number of larvae produced through the female function $W^{\text {ㅇ }}$ (this happens only in currently female individuals), and the number of larvae sired through the male function $W^{\widehat{\widehat{T}}}$ (this happens in males and in currently female individuals that inseminated other females before changing sex). The sum of these two components gives the total reproductive success of an individual $i$ :

$$
W_{i}=W_{i}^{\uparrow}+W_{i}^{\curvearrowright}
$$

In nature the breeding season of $C$. fornicata can extend over a long period ( 8 months in our sampling area, Le Cam 2009, p. 136), with ample time for females to produce several successive broods per year. In our experimental settings females produced on average one brood every 3 weeks, only a fraction of which was analyzed here (0-6 broods per female, see results). Hence we aimed to measure the "instantaneous" reproductive success of each individual rather than the total number of offspring parented, which would have been influenced by irrelevant experimental parameters (e.g. length of the experiment or date of death of an individual, and number of broods analyzed per female). Moreover, a few of the brood analyses did not produce a reliable estimation of the total number of larvae that they contained, because they were sampled too late after release

273 (and thus some larvae were lost through filtration by the adults). For these reasons we defined estimators of average reproductive success that were independent of the length of the experiment and the number of broods sampled per female. 

defined as the average brood size of that female:

$$
W_{i}^{\uparrow}=\frac{1}{B_{i}} \sum_{b} N_{i b}
$$

279

where $B$ is the number of broods produced by female $i$ and analyzed in this study and $N_{i b}$ is the size (total number of larvae) contained in brood $b$ of that female. These numbers were directly obtained from the size estimates of larval releases and parentage analyses described above.

Reproductive success through the male function has several components. It depends on the number of successful mates (female partners that bear progeny), the fecundity of these mates, and the proportion of larvae fathered by each individual within each brood. Hence we define:

$$
W_{i}^{\widehat{\jmath}}=\sum_{j \neq i} P_{i j} W_{j}^{\text {क }}
$$

where $P_{i j}$ is the average proportion of larvae sired by an individual $i$ when fertilizing broods produced by female $j$, and the product $P_{i j} W_{j}^{+}$is thus the average reproductive success of individual $i$ with female mate $j$ (that is, the expected number of larvae parented by individual $i$ for an average brood of female j). Summed over all successful mates, this gives the total reproductive success of individual $i$ through its male function. Average father contributions $P_{i j}$ were estimated from the results of the parentage analyses.

These definitions of reproductive success for male and female functions give the average number of offspring parented per time unit, considering that one time unit $=$ the time needed to produce one brood. These definitions are unbiased with regards to the length of the experiment and the number of broods sampled, but they do not take into account any potential variation in the frequency of brood production among females (see results and discussion). Moreover, fitness components such as the size of the larvae and their survival are also not taken into account here. With these data we analyzed the instantaneous reproductive success of an individual, how it was 
partitioned into male and female functions, and how it related to the size of individuals and their social environment.

Determinants of reproductive success

The relationship between reproductive success and adult size was tested in both sexes using generalized linear models (GLM) with a log link function and quasi-Poisson error family. In Crepidula fornicata size effects were confounded with the effect of the position of individuals within a stack (Dupont et al. 2006; Proestou et al. 2008; Le Cam et al. 2009). We addressed position effects in two different ways. First we drew the distribution of mating frequencies as a function of the distance between mating partners (data observed in all stacks during the whole experiment) and compared that to the distribution that would be expected if distance would not have any effect. The latter was obtained by considering that a male could fertilize any female in its stack with equal probability. Second, we looked at the proportion of offspring sired by a male within a brood as a function of the distance between this male and the mother that produced the brood.

\section{Results of the empirical study}

Adult survival and growth

Survival of the 223 individuals that were reared for 251 days ( 25 stacks, see methods) was $78 \%$ at day 251 . The growth of the surviving individuals, expressed as the difference between final and initial shell length, is shown in Figure 2. Growth was markedly stronger in males than females, and growth rate decreased with initial size. A good empirical fit to the data was provided by a polynomial model that included initial shell size, sex, an interaction between initial size and sex, and a quadratic term for the effect of initial size (shown in Fig. 2). This ad-hoc model predicts that a male grows twice as fast as a female of similar size. 
females and 31 males (sex at the end of the experiment; excluding one small individual that was lost during the experiment and two additional individuals for which the sex was uncertain due to a loss of raw data for stack 7 , see table 1). Parentage analyses were based on larval releases sampled between February 4 (date of the first observation of larvae) and May 20 (i.e. days 23-128). In this 106 day period we sampled 62 larval releases, corresponding to between 6 and 9 of the first batches of larvae released by each of our eight focal stacks (table 1). These batches contained between 2403 and 38860 larvae, with standard errors for these estimates in the range 160-2990. larvae, 3229 of which (99.7\%) could be unambiguously assigned to their two parents (including several reconstructed father genotypes that were not found in the adult set, for example because they were dead before the stacks were sampled in the field). These analyses indicated that nine larval releases appeared to be a mixture of 2 broods from distinct females of the same stack. Conversely, on three occasions two successive larval batches appeared to stem from the same brood (larvae released within a short interval, i.e. in the order of hours). Over the entire experiment, parentage data was obtained for between 5 and 11 broods for each stack (total 69 broods, table 1), with an average of 46.7 larvae successfully genotyped per brood. With these data we calculated the reproductive success of males (male function only) and females (female function + delayed male function).

Out of 31 males, 7 did not achieve any successful mating. The other males successfully mated with up to 3 female partners (Fig. S2). These males sired between 0 and $100 \%$ of the larvae of a given brood (Fig. S3; 0\% corresponds to when a male did not sire any offspring of a particular brood but successfully sired at least one other brood of the same mother). These numbers remained 
similar when averaged over broods of a specific female: the average proportion of offspring $P_{i j}$ sired by male $i$ with a given female $j$ ranged from 0.6 to $100 \%$ (median $27 \%$, mean $35 \%$ ).

$W^{\widehat{\lambda}}$ varied between 0 and 25761 offspring (average 5441). Six males out of 31 were excluded from this computation because they sired offspring with at least one female whose average reproductive success $W^{\uparrow}$ was not estimated (see below). We did not calculate the reproductive success $W^{\hat{\jmath}}$ of these males (even though we knew their success with other females) because it would have been underestimated.

Females may combine success through the female and male functions (via sperm stored and used by other females). Out of 37 females, 2 did not have any mates. Others successfully mated with up to 8 partners that may have been either males or females (Fig. S2). The reproductive success of these individuals is detailed below according to sexual functions. were not found to incubate any larvae when the stacks were dismantled). We considered that these females had no reproductive success through the female function $\left(W^{\circ}=0\right)$. number of larvae for only 44 out of the 69 broods that were genotyped (table 1, distribution of brood size shown in Fig. S4) because the remaining broods were sampled too late after release (see methods). These 44 broods were produced by 24 females, with 1 to 5 brood size estimates per female (average 1.83), yielding an estimate of average fecundity for each of these females $W^{\circ}$ in the range $2458-26400$ (average 14063, reduced to 11250 when the 6 females with no reproductive success were included). The seven remaining females produced only broods from which the total 
number of larvae could not be properly estimated. We have no estimate of reproductive success through the female function for these individuals (although we know which males they reproduced with, and in what proportions, from the genotypes of their offspring).

\section{Delayed male function}

Nearly half of the females (17 out of 37) contributed offspring through the male function. Nine of them fathered offspring with a single other female and the remaining 8 fathered offspring with 2 females. Just like their male counterparts, they sired between $0 \%$ and $100 \%$ of the offspring of a given brood and average proportions $P_{i j}$ ranged between 0.6 and $100 \%$ (median 32\%, mean 44\%). We were able to estimate the reproductive success through the male function for 12 of these 17 females: $W^{\widehat{3}}$ ranged 350-21235 offspring (average 8195, average including the 20 females that had no success through delayed male function 3073).

The resulting total reproductive success for females (that is, combining both female and male functions) is shown in figure 3. Females produced from 0 to 32121 offspring (average 15438).

Total reproductive success and its relationship with shell length

The average total reproductive success of females was nearly 3 times higher than that of males (15438 vs 5441 offspring; the difference is 10 -fold if one compares medians). Note that this is only possible because of sperm storage: many offspring had a female mother and a female father. The average reproductive success of females is greater because it combines female and male functions. The reproductive success of females with a male function was on average about $28 \%$ higher than that of females without a male function (17703 vs 13880).

Figure 4 shows the relationship between reproductive success and individual size in males and females (size at the onset of the experiment). Male shell length ranged from 7.6 to $51.3 \mathrm{~mm}$ while female shell length ranged from 35.6 to $57.3 \mathrm{~mm}$. The reproductive success $W^{\hat{\jmath}}$ of males smaller than $22 \mathrm{~mm}$ was nil, and it increased with male size ( $G L M, p=0.025)$. Female success through the 
female function $W^{\circ}$ or through both male and female functions $W^{\circ}+W^{\hat{\jmath}}$ did not significantly increase with female size (GLM, $p=0.68$ and 0.786 , respectively), although we note that the power of this analysis was limited by the restricted range of female sizes, in addition to the intrinsic uncertainty associated with fecundity estimates.

Other determinants of fitness

The vast majority of mating observed during the experiment ( $n=145$, Fig. S5A) happened between individuals that were directly in contact (distance $=0$, frequency $=40 \%$ ) or separated by only one other individual (distance=1, frequency=42\%), although one mating was observed while the male was separated from the female by as many as 6 individuals. This distribution differed clearly from expectations of random mating within each stack, where mating at distances of 0 to 3 intermediate individuals should represent ca. 15\% each (Fig. S5A).

Within a given brood, the proportion of larvae sired by a male appeared strongly constrained by the distance between this male and the mother of the brood (Fig. S5B). These proportions ranged 0 to $100 \%$ at distances of 0 and 1 and decreased strongly at larger distances. However, some outliers corresponding to two males that fathered a large fraction of the offspring of a distant female were identified (Fig. S5B).

Solitary mobile adults

As mentioned in the introduction, small individuals have the capacity to move and, in the field, some males may use this ability to get closer to females and perhaps to fertilize females from different stacks. During the experiment we observed 30 individuals (that is, 10\% of the 294 individuals from the 33 stacks reared in the lab) that moved away from their initial position. Only one of them changed position within its stack. That individual left its position at the top of a stack of eight individuals and adopted a secondary position on the side of the fourth individual. All other movements observed were rather surprising: 29 individuals left their original stack (as early as day 
14) and attempted to leave the plastic structure in which each stack was contained. They could not

419

420

421 succeed because it would have meant going above water (Fig. 1), but none of them came back on the stack. They remained on the plastic walls, sometimes halfway above water, for the rest of the experiment. We will refer to these individuals as "solitary".

These individuals were found to be males when they were sexed except for two individuals that were in the process of changing sex and one other that was immature. All of these solitary individuals were either initially positioned on the side of another individual (secondary position) or at the top of a stack (including some individuals that were initially $2^{\text {nd }}$ or $3^{\text {rd }}$ from the top before males above them moved away). Their average size was $25.2 \mathrm{~mm}$ (range 9.3-38.8) at the onset of the experiment. The movement of these individuals made it difficult to keep track of their identity throughout the experiment, so that we know both the initial and final size with certainty for only eight of them. They were reared for a variable period of time; hence we estimated their growth rate per day, for comparison with the growth of others. The shell length of these eight solitary individuals grew at an average rate of $0.008 \mathrm{~mm}$ per day. The average growth rate of all other males in the same size range $(9-40 \mathrm{~mm}, \mathrm{n}=53)$ was $0.031 \mathrm{~mm}$ per day.

We obtained reproductive success measurements for 6 such solitary individuals (included in the overall fitness estimates produced above for males). Surprisingly, each of them successfully fathered larvae during the experiment. They successfully sired offspring from one $(n=4)$ or two females $(n=2)$, and the average proportion of offspring $P_{i j}$ sired by solitary male $i$ with a given female $j$ ranged from 1.4 to $100 \%$ (median $32 \%$, mean $42 \%$ ). As a result, they achieved a reproductive success $W^{\hat{\jmath}}$ of between 240 and 10335 offspring (mean 4534).

\section{Model}

Empirical results from this study suggested that sperm storage might favor individuals that change sex at an earlier time than predicted from the size-advantage hypothesis because once a 
male has inseminated several females, further gain in reproductive success may be limited (see discussion). However, this effect is predicted to depend on the dynamics of sperm replacement in the sperm storage organs of females. In order to investigate these ideas we developed a simple model of reproductive success for a sequential hermaphrodite species with or without sperm storage. Here we outline the model framework, and a more detailed description is given in the online supplementary material.

Model outline

To investigate the effect of sperm storage on the timing of sex-change we simulate the reproductive success of an individual (ind. number 3 in Fig. 5) that interacts with one male and two females. Time is divided into discrete units and each female in the system produces one brood per time unit. Our focal individual starts its benthic life at size $L=0$ and grows at each time step. We then calculate the reproductive success that this individual would have as a male or a female at each time step, with or without sperm storage.

In the absence of sperm storage, the reproductive success of our focal individual as a male $\left(W^{\hat{}}\right)$ is determined simply by the fecundity of its female partners (brood size $N$ ) and the fraction of sperm $\varphi$ that our focal male provides to inseminate these females. With two females we have $W^{\hat{\delta}}=2 \varphi N$ at any time step and the male function stops as soon as the focal individual becomes female. In contrast with the male function, reproductive success through the female function depends on the size of the focal individual, following the size-fitness relationship reported by Li and Pechenik (2007). with the sex-change occurring when the individual reaches a size at which its reproductive success as a female becomes superior to its reproductive success as a male (in agreement with the sizeadvantage model, Ghiselin 1969). Sperm storage modifies reproductive success by allowing a female 
to continue fathering offspring for some time after sex-change, thus combining fitness output through male and female functions. However, success through the male function may decline rapidly, depending upon the dynamics of sperm reserves in inseminated females. We consider that females have sperm reserves that contain a proportion $p$ of sperm from our focal male and the reproductive output of our focal individual through the male function at any time step $\mathrm{t}$ is $W_{t}^{\hat{\widehat{C}}}=$ $2 p_{t} N$. If the sex-change has not happened, the focal individual is a male and we assume that it produces sperm continuously so that the fraction of its sperm in females' reserves is equal to the fraction of sperm $\varphi$ that our focal male provides to inseminate these females. After sex-change, we consider two situations (referred to as models 1 and 2 in Fig. 5). First, our focal individual (now female) stops producing sperm and thus the proportion of its sperm in the reserves decreases at some rate $m$ following $p_{t+1}=p_{t}(1-m)$. In that case its reproductive success through the male

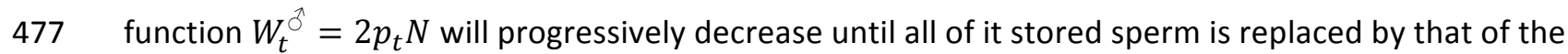
other male. In a second situation, the remaining male (number 4 in Fig. 5) shifts its mating effort toward the newly available female as soon as our focal individual has changed sex. To illustrate this point we consider the extreme situation where the top male no longer contributes sperm to the most distant female (note that this extreme situation is quite realistic considering the effect of distance between mating partners in our empirical results, see distance $=2$ in figure $S 5 \mathrm{~A}$ ). In that case the proportion of sperm from our focal individual that is stored in female 1 remains constant after the sex-change, because the female storage organ is not refilled by any male. Only in female 2 does the proportion of sperm from the focal individual decrease at rate $m$. The reproductive success (through the male function) of our focal individual after the sex-change thus becomes $W_{t}^{\hat{}}{ }^{\hat{1}}=\varphi N+$ $p_{t} N$.

To test for the effect of sperm storage we considered a baseline scenario (Fig. 6) where the parameters $\varphi$ and $N$ were held constant and we varied only sperm mortality $(m)$ and the timing of the sex-change. With this scenario the focal male sires $60 \%$ of the offspring produced by each female 
$491(\varphi=0.6)$ and female fecundity is set to $N=14000$ so that $W_{t}^{\widehat{\delta}}=16800$ offspring (grey solid line in

492 Fig. 6). If our focal individual is a female throughout its life, its reproductive success $W_{t}^{\dagger}$ varies from 0 at time $t=0$ to ca. 20700 offspring at time $t=100$ (black line in Fig. 6). Under these conditions the strategy that maximizes the reproductive value of the focal individual is to switch from the male to the female sex at time $t=47$, when $W_{t}^{\uparrow}$ gets larger than $W_{t}^{\hat{0}}$. The optimal strategy is highlighted with a thick grey line in figure 6 . Sperm storage changes this prediction by allowing a female to continue fathering offspring for some time after the sex-change, thus combining fitness output through male and female functions. An example is given in figure 6 , where sex-change occurs at time $t=20$ (compare reproductive success with and without sperm storage, shown by the dotted line and the dashed line, respectively). We determined the optimal timing of the sex-change (that is, the timing that maximizes lifetime reproductive output) with different values of sperm mortality $m=0,0.01$, $0.05,0.1,0.2$, and 0.3 .

To further understand the effect of sperm storage we calculated the lifetime reproductive output $\omega^{t}$ of an individual that changes sex at time $t$ (with sperm storage occurring) and compared it to the reproductive output $\omega^{*}$ of an individual that follows the optimal strategy for a situation where there is no sperm storage (i.e. sex-change at time $t=47$ ). The difference $\Delta \omega=\omega^{t}-\omega^{*}$ is equal to the net difference in lifetime reproductive output between these two situations and can be visualized in figure 6 as the area between the dotted line and the thick grey line. We calculated $\Delta \omega$ for the different values of sperm mortality $m$ listed above and with sex-change occurring at time $t=10, t=70$, and at every 5-generations interval between these two limits. strategy in absence of sperm storage has an expected lifetime reproductive output of $\omega^{*} \approx$

$5141.84 \times 10^{6}$ offspring. Sperm storage increases this lifetime reproductive success in nearly all situations (Fig. S6), except when sex-change occurs very early and sperm mortality is high $(t<25$, 

$m>0.05)$. However, it does not necessarily mean that the optimal timing of sex-change is altered by

517 sperm storage. The moment when changing sex yields the highest lifetime reproductive output is 518 shown in Figure 7 (also visible as the mode of each curve in Fig. S6). The model indicates that earlier 519 sex-change is favored only when sperm mortality is quite low $(m<0.1)$. This prediction changes if sperm proportions remain constant in one of the females (model 2). In that case, the highest lifetime

521 reproductive success will be obtained for an earlier sex-change in all conditions (Figs. 7 and S7).

522 
Three notable patterns emerged from our reproductive success measurements on male and

526

527

female slipper limpets: 1) male success increased strongly with size, 2) sperm storage was a remarkably strong driver of reproductive success, and 3) small mobile males were identified, and they had a significant level of reproductive success is. We will consider these results in light of the size advantage theory for protandrous sex-change.

\section{Size-fitness curves in Crepidula fornicata}

The reproductive success of females was on average $50 \%$ greater than that of males in the same size range (i.e. all individuals with shell length $L>35.5 \mathrm{~mm}, \mathrm{n}_{\text {males }}=9, \mathrm{n}_{\text {females }}=27$, average male success=10433 offspring, average female success=15438). This indicates that changing sex is beneficial in terms of immediate fitness. In agreement with the size advantage hypothesis, individuals change sex at a condition (linked with size or age) that allows them to produce more larvae through their own fecundity than they would sire as a male.

Remarkably, this result was obtained despite the fact that male success increased sharply with size, confirming results from Dupont et al. (2006) and Proestou et al. (2008). While we do not know whether male body size has a direct effect on reproductive output (e.g. through variations in sperm production), size is correlated with the position of males within a stack and this has a critical influence on reproductive success ( Dupont et al. 2006; Proestou et al. 2008; Le Cam et al. 2009, this study). Due to the stacking process, males find themselves closer to females as they age/grow, and we found this to be a primary determinant of the male size-fitness curve. The two major components of male reproductive success, that is, mating success and the proportion of offspring sired within a given brood, are both strongly affected by the distance to females. More than $80 \%$ of all our mating observations occurred between individuals in direct contact or separated by only one other individual (Fig. S5A), and the male in such matings is expected to sire on average nearly half of 
the offspring of a given brood (Fig. S5B with distance to female=0 or 1). However, this advantage of large-males does not outweigh the gain in immediate fitness expected from the sex-change, since females in this study had a greater average reproductive success. The situation found in Crepidula fornicata therefore illustrates that changes in male reproductive success with size (here due to the species' social system) are compatible with protandry, as expected from theory.

In contrast to males, a female's reproductive success depended almost exclusively on her own fecundity. With the exception of solitary females (which occur when juvenile limpets are not in contact with conspecifics and rapidly adopt the female sex, e.g. Coe 1938), all females in the wild have at least one potential mating partner, and we found that above one the number of mating partners has no effect on a female's total reproductive success (not shown). Female fecundity has been shown to be linked with size (Hoagland 1978; Richard et al. 2006; Li and Pechenik 2007; Proestou et al. 2008). However, here we did not observe this relationship because the females in our stacks had a very limited size range (they were all rather large females) and perhaps also because of the uncertainty in our fecundity estimates. Yet we found that female growth was much less rapid than male growth (Fig. 2), confirming previous findings by Collin (1995). Hence very little additional gain in reproductive success is to be expected once an individual has changed sex (at least in the size growth.

Bringing together these observations for male and female reproductive success, we found that large individuals had an advantage when they were female but that there was no clear advantage for small individuals in being male. Our data indicate that small males have very little or no reproductive success. Based upon two previous studies that reported quantitative relationships between shell length and fecundity, expressed as the number of offspring (Li and Pechenik 2007; Proestou et al. 2008), small males from our study would have been predicted to have had the same or greater 

being a male is a fitter option than being a female at any point during an individual's life. What is important for the evolution of sequential hermaphroditism, however, is the trajectory of sex-specific reproductive values, rather than immediate success. The faster growth of males is one factor that will create a difference in reproductive values between sexes. Because there is no clear immediate advantage in being male at any point in life, we conclude that the faster growth rate of males is an important factor that will favor protandry, by allowing individuals to rapidly reach a size that confers high female-fitness. That conclusion however needs to be pondered in light of the effect of sperm storage and accrued mobility of small males. is particularly interesting from the females' point of view: nearly half of the females contributed offspring through the male function, and these females had an average reproductive success about $28 \%$ higher than the others. This seems to have important consequences for the timing of the sexchange, because without this fitness component the advantage of being a female is reduced (compare the dotted and dashed lines in Figure 4). Females that did not father any offspring had an average reproductive success of 13880 offspring, compared with 10433 for males in the same size range. This difference is rather small given the number of individuals for which we could estimate reproductive success and the number of broods on which each of these estimates was obtained. Because these males monopolize most mating opportunities one can imagine that the sperm storage organs of the females below them are filled mostly with their sperm. If further sperm transfer has diminishing returns in terms of the proportion of offspring sired in upcoming broods, then it would 
be beneficial to switch to the female sex earlier than predicted from body size. This benefit, however, is likely to be temporary, depending upon the duration of sperm storage, direction of sperm precedence, and the modalities of sperm replacement.

Modeling the simplest possible system, we found that sperm storage alone does not automatically promote an earlier sex-change. In many situations the fitness benefit obtained through sperm storage is highest at the same moment as the optimal timing of sex-change in the absence of sperm storage (Fig. 7). That is, sperm storage increases an individual's fitness but does not necessarily alter the timing of the sex-change that would maximal lifetime reproductive success.

This result holds as long as sperm replacement is rapid (i.e. $m>0.05$ in Fig. 7). If sperm is lost or replaced at a slower pace, then sperm storage can result in earlier sex-change because under these conditions the delayed male function persists throughout a female's lifetime. Sperm viability and replacement rate are thus key factors, as was shown to be the case in the context of sex allocation in simultaneous hermaphrodites (e.g. Charnov 1996). Empirical estimates, however, are difficult to obtain. Using our parentage data, we found one situation where a ballpark figure for our parameter $m$ could be estimated (Fig. 8): the female at the base of stack 1 (individual 1 in figure 8) repeatedly produced broods partly sired by the second female of that stack (individual 2). That is, individual 2 was already a female when the experiment began, but its sperm stored in female 1 was repeatedly used to fertilize that female's eggs. Four consecutive broods produced by female 1 were analyzed for this study (16 to 19 days between successive broods). We genotyped and analyzed one additional brood produced 180 days later by the same female ( $n=87$ larvae). A decay model fitted to these data (see supplementary methods and figure 8 ) gave an estimate of $m=0.14$. This rough estimate falls into the region where sperm storage might not have any effect on the timing of the sex-change, under the conditions of our model. While this estimate illustrates only one situation (namely, a fourindividual stack where the female father that we looked at was in competition with two other potential fathers), it suggests that sperm storage could have little effect on the timing of the sex- 
change if stacks generally contain a small number of individuals (because in such stacks females would never be far from males and they would receive new sperm continuously).

A situation where sperm storage would favor an earlier sex-change, even for high rates of sperm mortality, would occur when the sex-change prompts the remaining males in the stack to shift their copulation effort towards that new female (model 2 in Fig. 5). In that case sperm competition would be relaxed in the older females because they would receive less sperm (possibly none). Previous sperm donors would thus benefit from sperm storage for longer periods of time.

Parentage analyses from our empirical study revealed at least one situation where sperm storage must have benefited one father over a long time period. The female at the base of our largest stack (stack $8, n=15$ ind., including 6 females) produced two broods that we analyzed. With the exception of one larva (out of 47), the first brood appeared to be fertilized by sperm from a single female (third individual in the stack, starting from the base). The second brood, produced three weeks later, gave the same result (46/47 larvae fathered by female 3 ). Because the first six individuals of this stack were females at the end of the experiment, it is likely that the basal female had not been receiving any sperm for a long time, giving female 3 a long term advantage ( $m$ was effectively equal to 0 in the three week interval between the two broods that we analyzed). Such a pattern may be common in the wild: a nine-year long monthly survey of our source population showed that $25 \%$ of the stacks contained 4 females or more ( $n=3693$ stacks analyzed, S. Le Cam, F. Riquet, F. Viard, unpublished data).

Finally, we note two additional effects of sperm storage that have a bearing on sex-change. First, sperm storage reduces the immediate cost of changing sex to nearly zero. Second, females with little or no access to males (e.g. in stacks containing more than 4 or 5 females) can continue to produce offspring as long as their sperm stores are not emptied. We conclude that sperm storage might influence the timing of the sex-change in protandrous species with internal fertilization (e.g. all calyptraeids), a topic that deserves a more detailed theoretical treatment. Empirical research 
focusing on processes of sperm viability and competition (including sperm precedence and postcopulatory sexual selection) would also be particularly informative.

Small mobile males: an alternative strategy, or is it?

It was quite surprising to watch small males (range 9.3-38.8 $\mathrm{mm}$ ) rapidly moving away from their stack and remain isolated for months without any attempt to return to their original stack. It was all the more surprising to realize that all six such individuals that were included in our fitness measurements actually had successfully sired a rather large quantity of offspring (average reproductive success estimated to 4534 offspring, which is just below the average for all the other males, 5727). Had these males successfully integrated a new stack, it is very likely that they would have achieved better reproductive success. Note that sperm storage is fundamental to this strategy.

These results therefore settle the debate as to whether small mobile males exist, and whether these males father a significant number of offspring. This behavior does exist, and it explains why a fraction of offspring were assigned to unsampled males in all the paternity analyses that have been performed so far with Crepidula fornicata stacks (Dupont et al. 2006; Proestou et al. 2008; Le Cam et al. 2009, this study). The fitness of small mobile males was no better than that of large males that were in direct contact with females (as wondered by Charnov 1982), but neither was it markedly worse, and fitness could be further increased if small mobile males were able to rove freely from stack to stack, an hypothesis that remains to be tested. Based upon our data, the advantage conferred by mobility to small individuals did not alter the general pattern of increase in male reproductive success with size (solitary males were included in all calculations). But these data ignore the success that mobile males could have had in neighboring stacks.

An interesting question is left unanswered: does the behavior of the small mobile males represent an alternative strategy (see reviews e.g. in Taborsky et al. 2008; Neff and Svensson 2013), 
in which individuals would engage in exploratory movement, grow more slowly, and perhaps change

672

673

674

675

676

677 sex later or not at all, or do most or all individuals adopt this behavior when they are young before associating with a stack and waiting for their turn to become close to the females and eventually change sex? The fact that many of the solitary males observed in this study were actually first observed on top of a stack (in some cases after the male above them had left) suggests that males may pile up and then temporarily leave their position at some point to rove down the stack towards the females or try to reach other stacks. Also in favor of the second hypothesis, it seems unlikely that the reproductive value of an individual that remains a small male for all of its life would exceed that of a male that grows up quickly and at some point monopolizes fertilization with one or two females and then switches to the female sex. The mobility strategy could pay off, however, in very dense populations with large stack sizes, a situation that has been observed in some areas in the introduced range of Crepidula fornicata. The density of the population where our adults were collected was estimated twice per year in 2002, 2003, and 2004 by L. Dupont and colleagues (Dupont 2004 p. 69), yielding an average density of 21 ind. $\mathrm{m}^{-2}$, although with a very patchy distribution (maximum local density $=201$ ind. $\mathrm{m}^{-2}$ ). It seems possible that mobile males could fertilize females from distinct stacks under these conditions.

Limits of this study

One original contribution of this study in terms of the data produced is that reproductive success was measured from sequences of broods (up to 5 broods from a single female), and that a large number of larvae from each brood was analyzed (46.7 larvae on average). However, we could not estimate brood size in all cases, a frustrating limitation that led us to work only with the average reproductive success for males and females (calculated from a small number of broods per female) and prevented more detailed analyses (e.g. covariance between brood size and father identities). Notwithstanding our efforts to analyze multiple broods, the data presented here give only a 
snapshot of an individual's total reproductive output (Crepidula fornicata reproduces continuously

697

698

699

700

701

702

703

704

705

706

707

708

709

710

711

712

713 during an extended breeding season in the wild). Thus one main limit of this study is that it ignores several components of fitness. We do not know whether the frequency of brood production is variable among females, and, most importantly, if that variance is linked with size. There is no obvious reason why the development of larvae should be quicker for larger mothers, but such a relationship could alter some of our conclusions (e.g. regarding the female size-fitness curve). Furthermore, while our data are informative on the role of sex and size on the "instantaneous" reproductive success per brood produced, downstream components of fitness such as larval growth and survival remain unexplored (see Le Cam et al. 2009 for an effect of multiple paternity on the variance in larval growth).

Finally, as mentioned above, the success of males outside the stack where they were sampled is unknown. If male mobility between stacks is more extensive than currently thought, then it would be important to take extra-stack paternity into account to refine the male size-fitness curve. Male mobility between stacks could be relevant to female fitness as well, because mobile males could have some (currently unknown) reproductive success through delayed male function in neighboring stacks. In theory this point could be tested by genotyping all adults from within a certain radius in a moderately high density population, and seeking paternity for a sample of broods from incubating mothers.

\section{Conclusion}

Size-fitness curves established for male and female Crepidula fornicata were essentially in agreement with predictions from the size advantage hypothesis of sex change. Most importantly, females were large individuals and had a higher average reproductive success than males of equal size. On the other hand, the immediate advantage of being male for small individuals is less 
apparent, mostly because the mating system of Crepidula fornicata is advantageous to large males, as their size and/or position grant them both a higher mating success and fertilization success. We suggest that the higher growth rate of males, and thus the increased reproductive value of individuals which start their life as males, confers a decisive advantage to protandry in Crepidula fornicata. Male mobility might also contribute to the immediate reproductive success of small males if they succeed in copulating with females from distinct stacks, a hypothesis that is yet to be tested. We found that sperm storage affects strongly the reproductive success of males and females, and that it could theoretically promote earlier sex change in protandrous species. Missing information on the production of sperm, holding capacity in female organs, and sperm precedence and displacement processes are determinant in this regard. Overall, Crepidula fornicata provides an example of a protandrous species where male fitness increases quite sharply with age/size but protandry is still adaptive because large females have an even higher fitness, in part due to sperm storage effects.

\section{Acknowledgements}

We thank the following people for their input in this study through invaluable discussions, suggestions, or technical help: Mark Cock, Thierry Comtet, Claire Daguin-Thiébaut, Regis Lasbleiz, Sabrina Le Cam, Nicolas Perrin, Florentine Riquet, Denis Roze, Eric Thiébaut, and Jon Yearsley. Editors Susan Kalisz, Peter Nonacs, and two anonymous referees helped improve the manuscript. We are grateful to the divers of the Marine Operation Department of Roscoff biological station for sampling adult limpets in the wild and we thank the Biogenouest Genomics and Genomer plateforme facilities for technical support. This study was funded by Region Bretagne through a grant from the SAD funding program to TB. 
Andersson, M. 1994, Sexual selection. Princeton, Princeton University Press.

Angeloni, L., J. W. Bradbury, and E. L. Charnov. 2002. Body size and sex allocation in simultaneously hermaphroditic animals. Behavioral Ecology 13:419-426.

Charnov, E. L. 1982, The theory of sex allocation: Monographs in Population Biology, Princeton University Press.

-. 1996. Sperm competition and sex allocation in simultaneous hermaphrodites. Evolutionary Ecology 10:457-462.

Coe, W. R. 1936. Sexual phases in Crepidula. The journal of experimental zoology 72:455-477.

-. 1938. Conditions influencing change of sex in mollusks of the genus Crepidula. Journal of Experimental Zoology 77:401-424.

Collin, R. 1995. Sex, size, and position: A test of models predicting size at sex change in the protandrous gastropod Crepidula fornicata. American Naturalist 146:815-831.

-. 2013. Phylogenetic Patterns and Phenotypic Plasticity of Molluscan Sexual Systems. Integrative

Conklin, E. G. 1897. The embryology of Crepidula. Journal of Morphology 13:1-226. 35:12-15. 
Hoagland, K. E. 1978. Protandry and the evolution of environmentally-mediated sex change: a study of the mollusca. Malacologia 17:365-391.

Iwasa, Y. 1991. Sex change evolution and cost of reproduction. Behavioral Ecology 2:56-68.

Jones, O. R., and J. L. Wang. 2010. COLONY: a program for parentage and sibship inference from multilocus genotype data. Molecular Ecology Resources 10:551-555.

Kazancioglu, E., and S. H. Alonzo. 2010. Classic predictions about sex change do not hold under all types of size advantage. Journal of Evolutionary Biology 23:2432-2441.

Le Cam, S. 2009. Grégarité, changement de sexe et polyandrie : modalités de la reproduction chez une espèce invasive Crepidula fornicata, Pierre and Marie Curie University (Paris 6).

Le Cam, S., J. Pechenik, M. Cagnon, and F. Viard. 2009. Fast vs. slow larval growth in an invasive marine mollusc: does paternity matter? Journal of Heredity 100:455-464.

Le Cam, S., and F. Viard. 2011. Infestation of the invasive mollusc Crepidula fornicata by the native shell borer Cliona celata: a case of high parasite load without detrimental effects. Biological Invasions 13:1087-1098.

Li, W., and J. A. Pechenik. 2007. Effect of inbreeding on reproduction and juvenile performance in two marine gastropods with contrasting reproductive patterns. Marine Ecology Progress Series 346:219-234.

Michiels, N. K. 1998. Mating conflicts and sperm competition in simultaneous hermaphrodites, Pages 219-254 in T. R. Birkhead, and A. P. Moller, eds. Sperm competition and sexual selection, Academic press.

Munday, P. L., P. M. Buston, and R. R. Warner. 2006. Diversity and flexibility of sex-change strategies in animals. Trends in Ecology \& Evolution 21:89-95.

Neff, B. D., and E. I. Svensson. 2013. Polyandry and alternative mating tactics. Philosophical Transactions of the Royal Society B-Biological Sciences 368:11.

Orton, J. H. 1912. An account of the natural history of the slipper limpet (Crepidula fornicata). Journal of the Marine Biological Association of the United Kingdom 9:437-478. 
Pechenik, J. A., M. Blanchard, and R. Rotjan. 2004. Susceptibility of larval Crepidula fornicata to predation by suspension-feeding adults. Journal of Experimental Marine Biology and Ecology 306:75-94.

Proestou, D. A., M. R. Goldsmith, and S. Twombly. 2008. Patterns of male reproductive success in Crepidula fornicata provide new insight for sex allocation and optimal sex change. Biological Bulletin 214:192-200.

Richard, J., M. Huet, G. Thouzeau, and Y. M. Paulet. 2006. Reproduction of the invasive slipper limpet, Crepidula fornicata, in the Bay of Brest, France. Marine Biology 149:789-801.

Riquet, F., M. Ballenghien, A. Tanguy, and F. Viard. 2011. In silico mining and characterization of 12 EST-SSRs for the invasive slipper limpet Crepidula fornicata. Marine Genomics 4:291-295.

Taborsky, M., R. F. Oliveira, and H. J. Brockmann. 2008. The evolution of alternative reproductive tactics: concepts and questions, Pages 1-21 in R. F. Oliveira, M. Taborsky, and H. J. Brockmann, eds. Alternative reproductive tactics, Cambridge University Press.

Warner, R. R. 1988. Sex change and the size-advantage model. Trends in Ecology \& Evolution 3:133136.

West, S. 2009, Sex allocation: Monographs in Population Biology, v. 44, Princeton University Press. 


\section{Tables}

Table 1: Description of the eight stacks chosen for reproductive success analyses. We report the total number of individuals in each stack, the number of females, the number of larval releases analyzed per stack, and the number of actual broods identified from these batches after parentage analyses (see text). The number of broods from which female fecundity (i.e. brood size) could be estimated is given in the last column.

\begin{tabular}{cccccc}
\hline Stack & No. Ind. & No. females & No. larval releases & No. broods & No. brood size estimates \\
\hline 1 & 4 & 2 & 8 & 11 & 9 \\
2 & 5 & 3 & 6 & 5 & 4 \\
3 & 6 & 5 & 8 & 9 & 8 \\
4 & 8 & 4 & 8 & 8 & 5 \\
5 & 10 & 4 & 8 & 9 & 3 \\
6 & 11 & 6 & 9 & 9 & 4 \\
7 & 12 & $7^{\mathrm{a}}$ & 7 & 9 & 3 \\
8 & $15^{\mathrm{b}}$ & 6 & 8 & 9 & 44 \\
Total & 71 & 37 & 62 & 69 & 8 \\
\hline
\end{tabular}

a) The sex of two individuals from stack 7 was unknown due to a loss of raw data. The minimum number of females in this stack was 7.

b) Including one small individual $(10.6 \mathrm{~mm})$ that was lost during the experiment, probably during cleaning operations. This individual is not included in any of the analyses. 


\section{Figure legends}

Figure 1: Experimental set-up. Thirty-three stacks of 4 to 15 Crepidula fornicata sampled in the wild were kept in the lab. Each stack was reared in an individual structure allowing us to collect larvae as they were produced, without any mixing of larvae from other stacks. Photo credit: Wilfried Thomas, Marine Operation Department, Station Biologique de Roscoff.

Figure 2: Shell growth observed during the experiment for the individuals from 25 stacks that were kept alive in the lab for 251 days. Shell length at the start of the experiment was measured from living individuals in stacks, hence there is some uncertainty associated with such measures. Growth predictions from a polynomial model (lines) highlight the difference in growth rates between sexes.

Figure 3: Distribution of the reproductive success of adult Crepidula fornicata (24 males and 27 females from 8 stacks, that is, males for which an estimate of the male function was obtained and females for which estimates of the male and female functions were both obtained). Reproductive success is defined as the average number of offspring parented per brood produced (see text). Some individuals (black bars) that recently changed sex from male to female combine reproductive success through the female function (production of eggs) and the male function (male gametes produced prior to sex-change and now used by older females through sperm storage). Here we see that such females are frequent in the group of individuals showing the highest success (e.g. > 20000).

Figure 4: Reproductive success of adult Crepidula fornicata as a function of their sex and size. A female's reproductive success has two components: the number of larvae produced ("female function": empty black dots) and the number of larvae fertilized by male gametes produced prior to sex-change and stored by older females ("delayed male function"). The sum of these two components is shown by the solid black dots. Arrows show how this delayed male function (sperm storage) increases a female's fitness. The fitness of females does not increase significantly with their size, whether one considers only the female function (dotted line) or their total fitness (combining 
male and female functions, dashed line). Male fitness (solid grey dots) increases significantly with shell size (solid grey line).

Figure 5: Cartoon of the system considered for modelling the effect of sperm storage on reproductive success. We start with a situation where 2 males contribute sperm to 2 females at each time step in proportions $\varphi$ and $1-\varphi$. We follow the reproductive success of individual number 3 , which may change sex at some point. We consider two cases. In model 1 , sex-change of our focal individual results in male 4 contributing all sperm to females 1-3. In model 2, reproductive effort from male 4 will switch towards the newly available female, so that the most basal female does not get any sperm and relies only on stored sperm to produce larvae.

Figure 6: Example dynamics of reproductive success from the model 1 depicted in Figure 5, with or without sperm storage. The solid lines represent the reproductive output of the focal individual considered in our model if it remains a male (grey line) or a female (black) throughout its life. The strategy highlighted with a thick grey line shows the optimal strategy in absence of sperm storage (sex-change at time $t=47$ ). The dashed and dotted lines represent the reproductive success in case of sex-change at an earlier time $t=20$ without or with sperm storage, respectively. In this example $m=0.05, \varphi=0.6$.

Figure 7: Optimal time of sex-change as a function of sperm mortality rate $m$. In absence of sperm storage lifetime reproductive success is maximized with sex-change happening at time $t=47$ (dashed grey line). Earlier sex-change is predicted in a situation where sperm is stored and does not disappear too rapidly (model 1 , solid black curve) or is not fully replaced by sperm from other males (model 2, solid grey curve).

Figure 8: Decay of the proportion of larvae from stack 1 mother 1 sired by individual 2 (which was already female when the first brood was produced, here represented at time $t=0$ ). Each dot represent data from one brood from mother 1 , and grey bars show standard error of the proportion 
of larvae sired by individual 2 . The first four data points come from four consecutive broods, starting February 10, 2011, with an average interval of 18 days (= one time unit). The final brood was produced August 9, 2011 (exactly 180 days after the first one). The black line shows a model of the form $p_{t}=p_{0}(1-m)^{t}$, with best fit obtained for $p_{0}=0.21$ and $m=0.14$ (adjusted $R^{2}=0.93$ ). This observation suggests that the fertilization success of individual 2 with female 1 decreases at rate $m=0.14$. Under the simplest rules of sperm replacement (fair raffle, no sperm precedence, as in our model) it can be interpreted as a sperm mortality rate of 0.14 . This decay rate can also result from more complex, unknown, sperm replacement rules. 
Figure 1

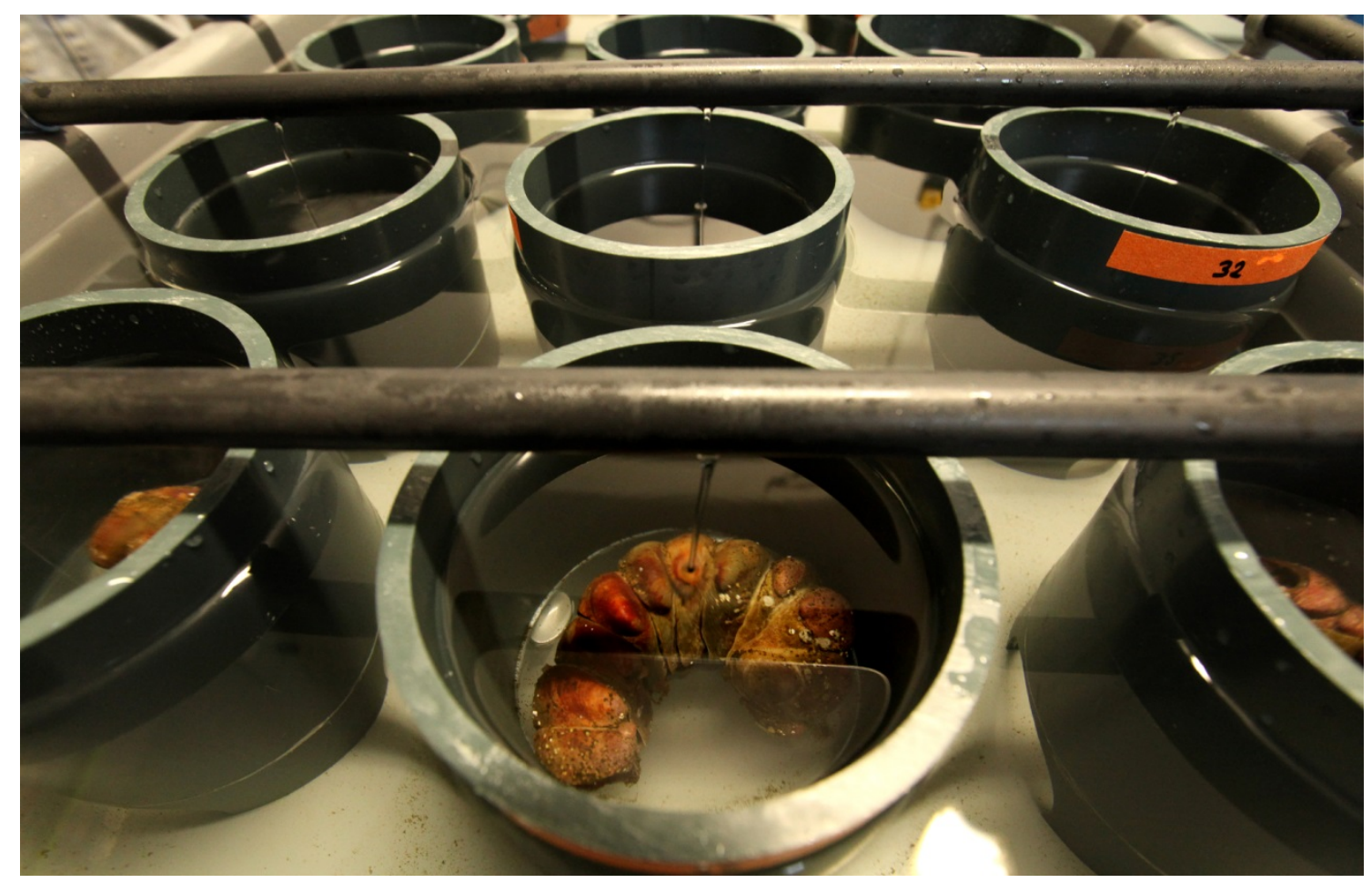


Figure 2

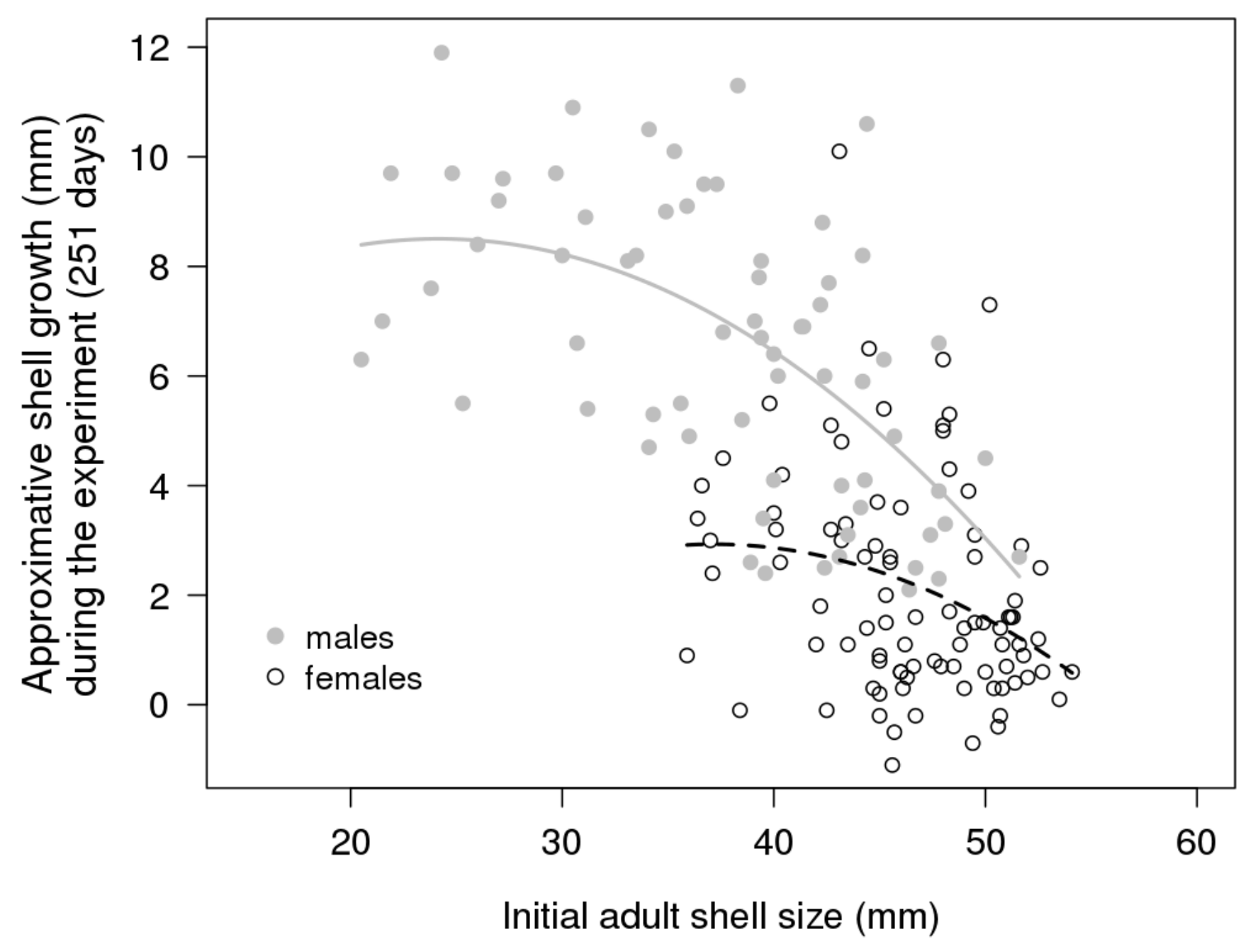


Figure 3

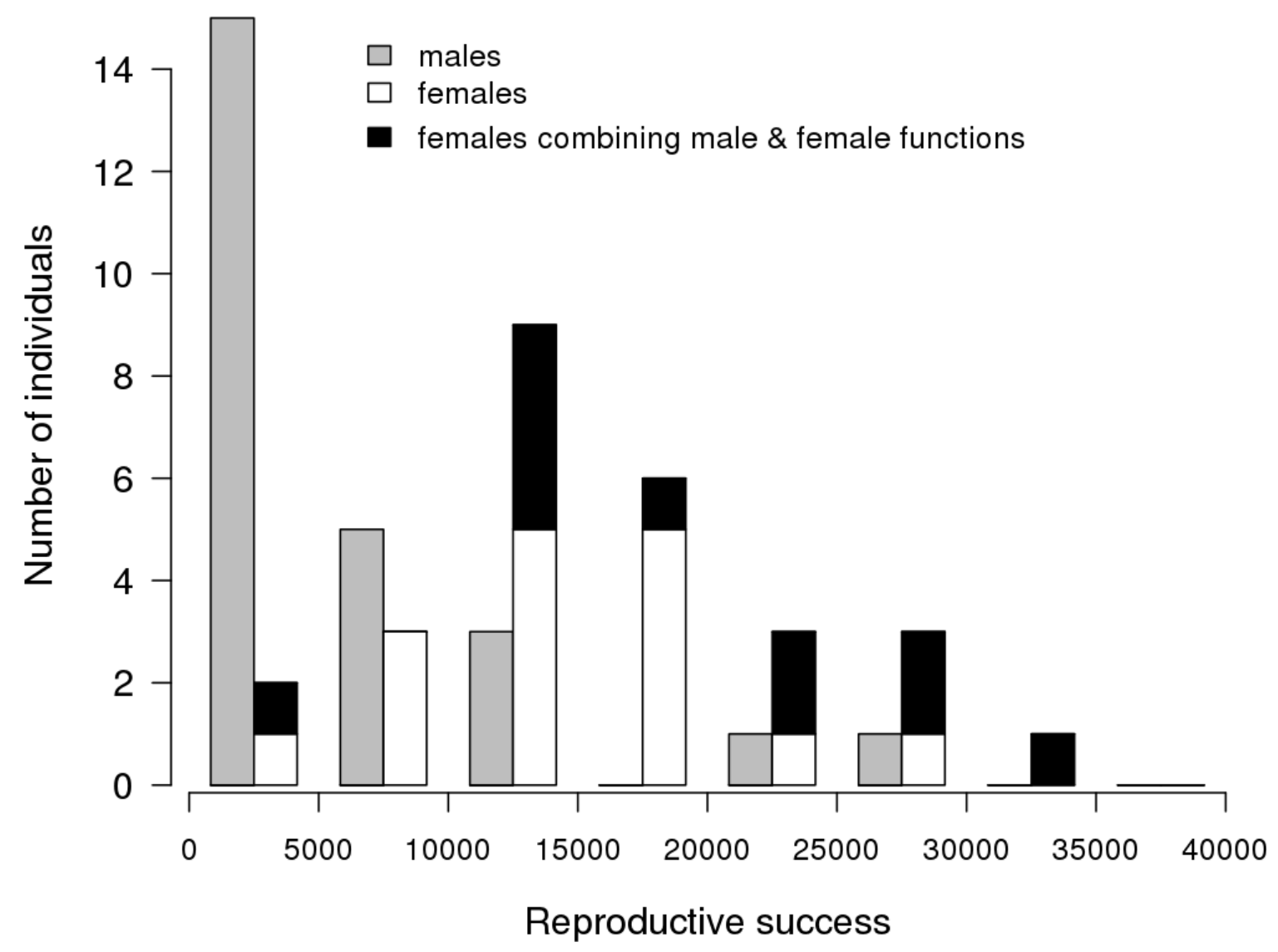


Figure 4

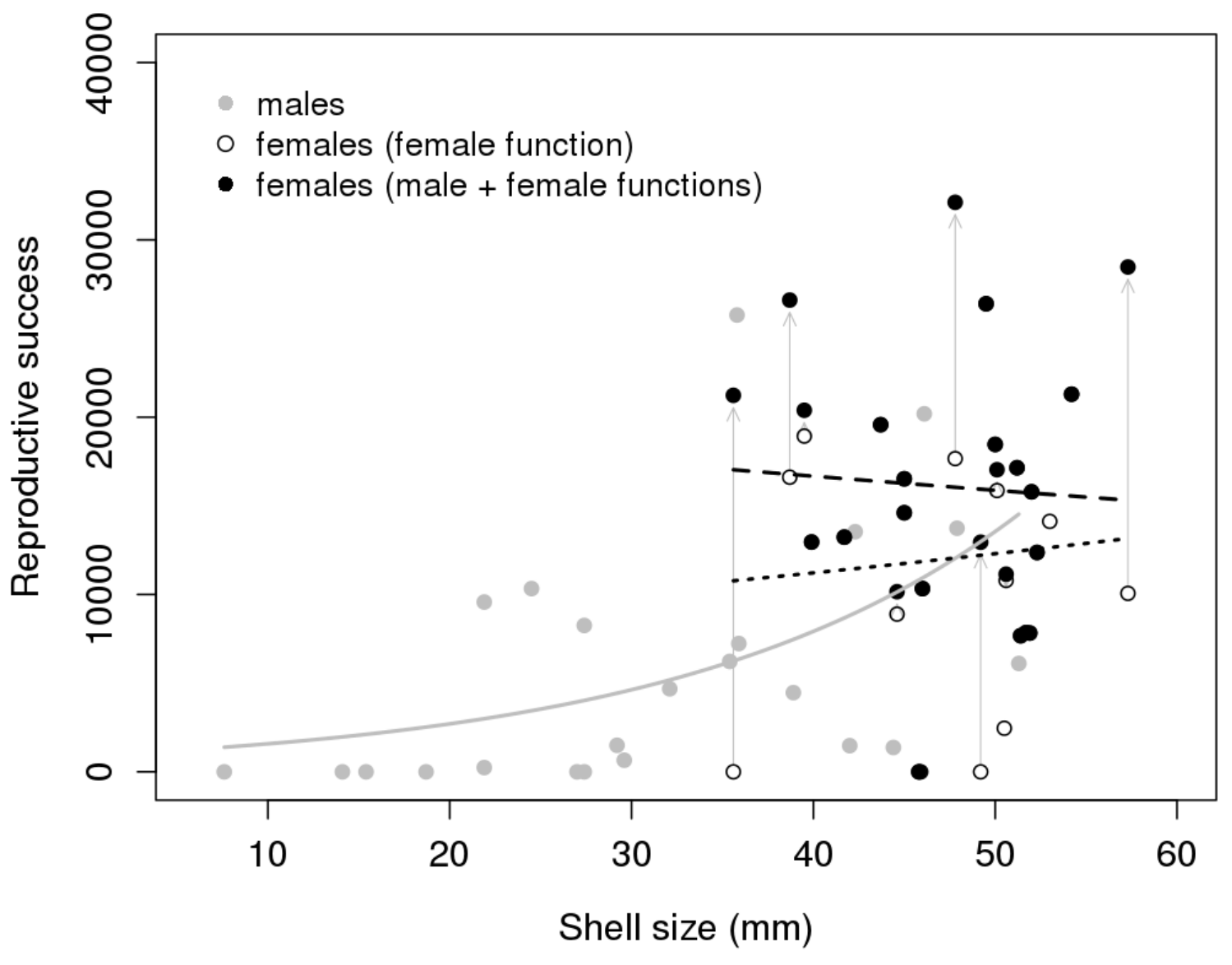


Figure 5

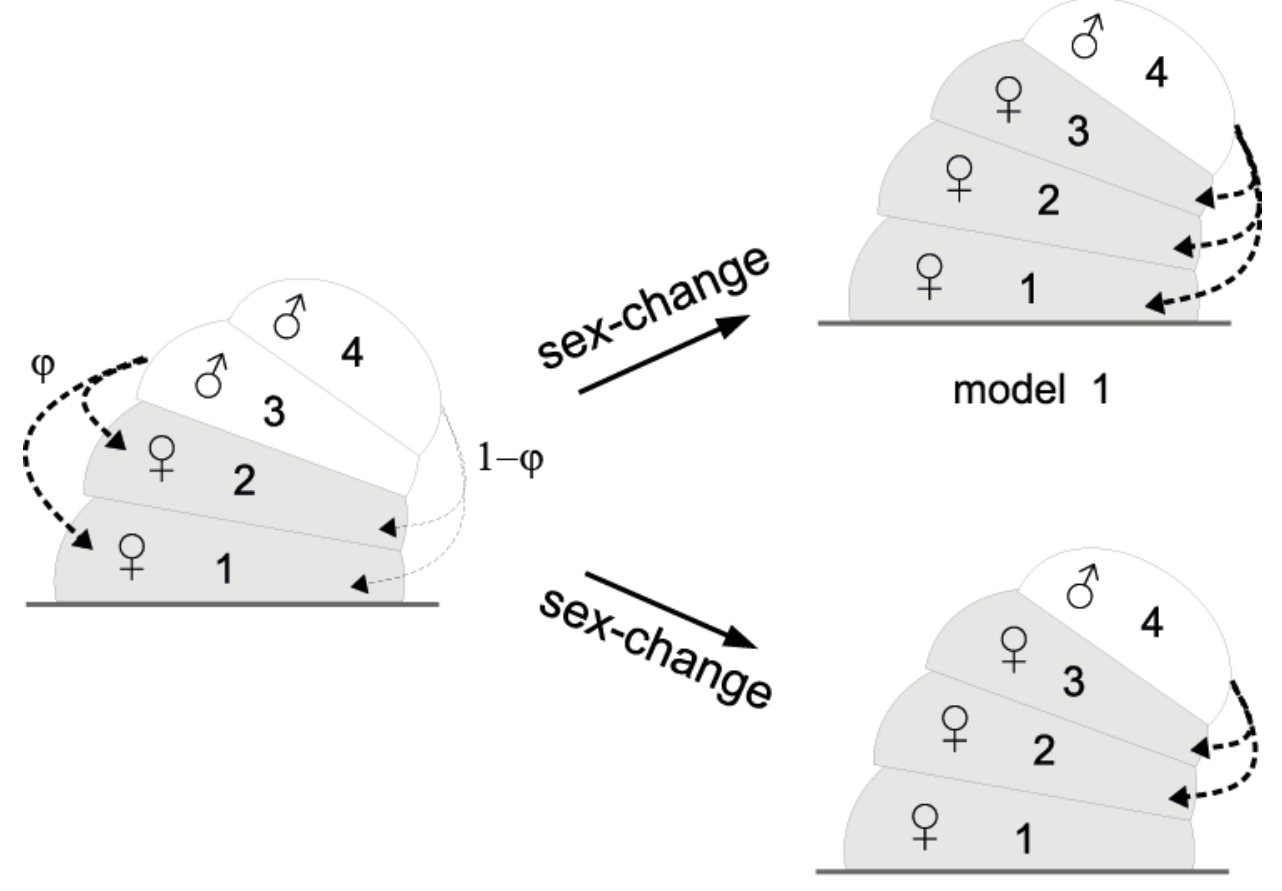

model 2 
Figure 6

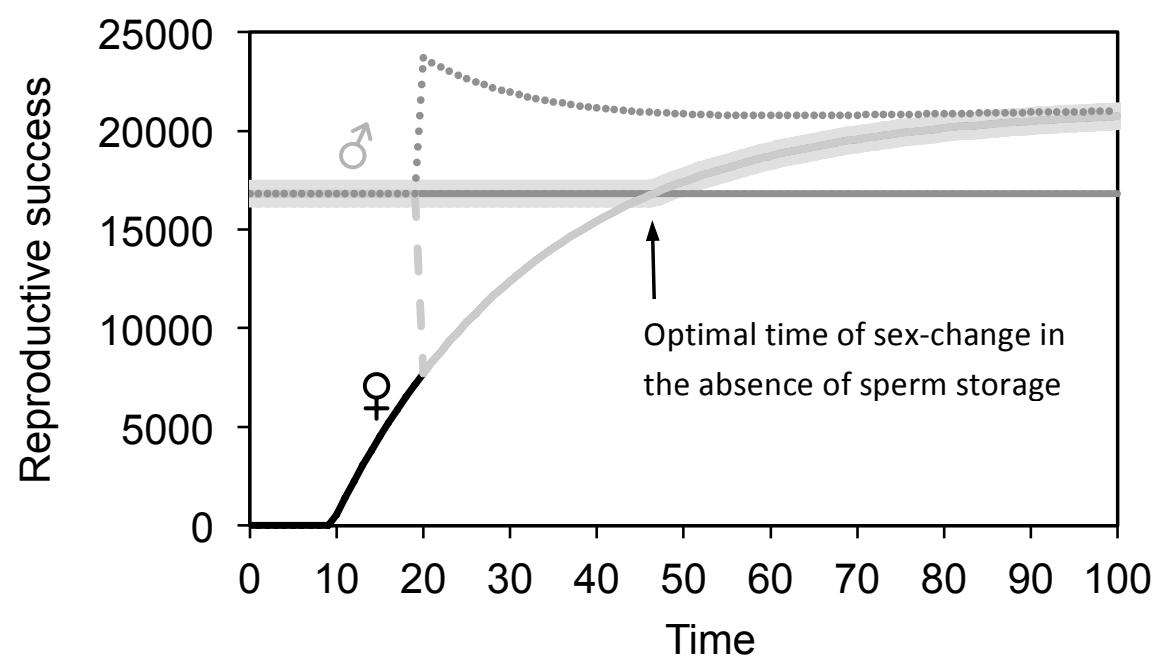


Figure 7

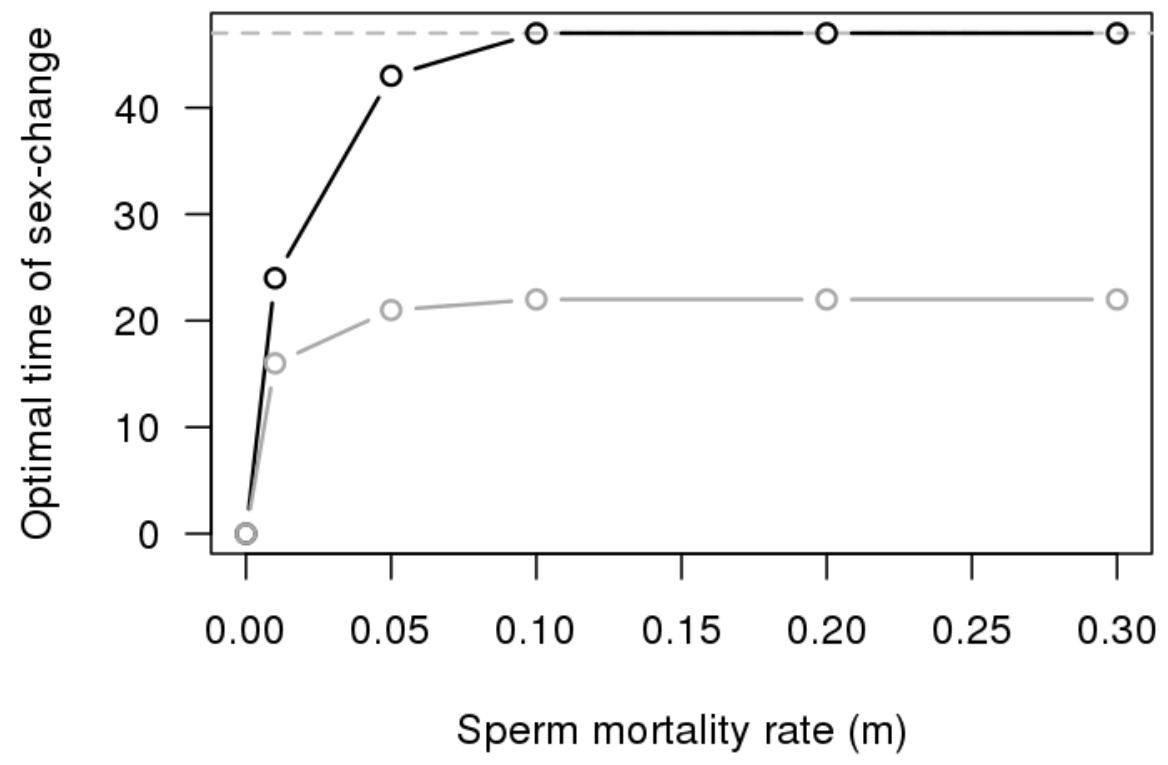


Figure 8

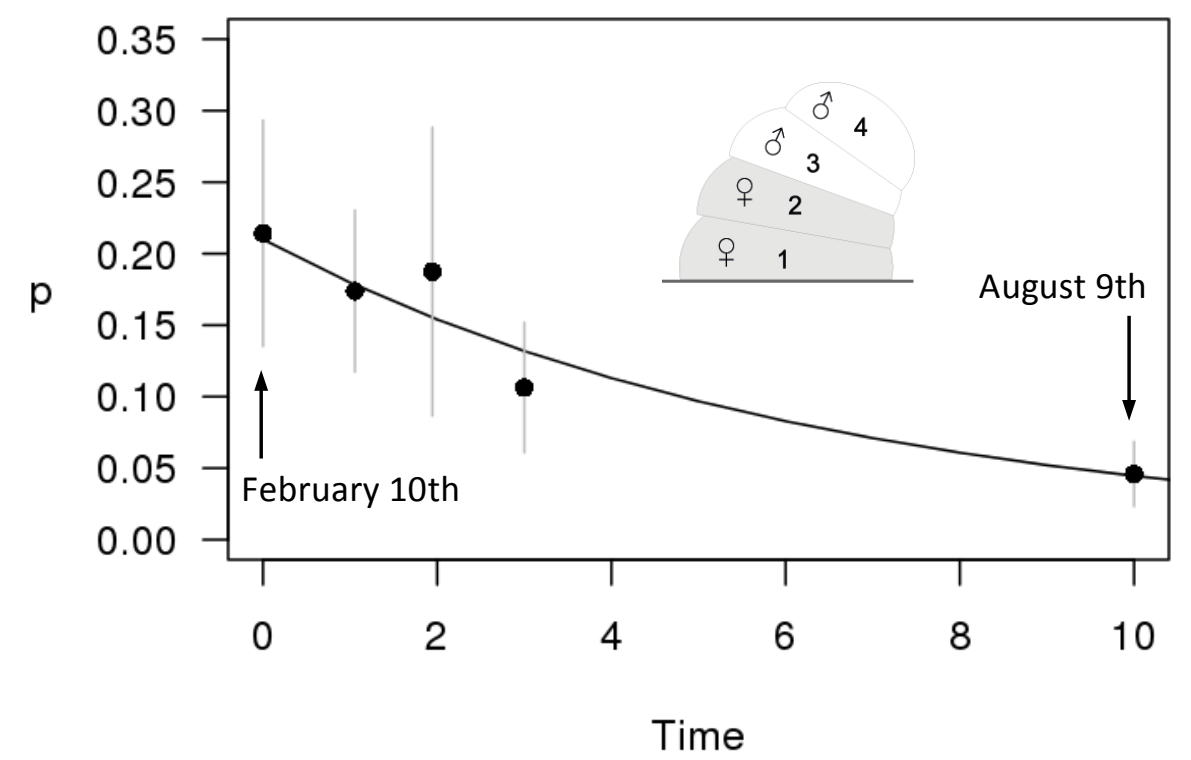


2

3

4

5

6

Experimental set-up

We kept all adult $C$. fornicata (33 stacks) in a common tray with circulating $5 \mu \mathrm{m}$-filtered

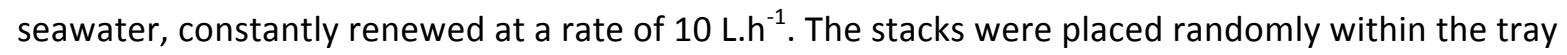
just after their collection in the wild. Seawater temperature was $9^{\circ} \mathrm{C}$ at the collection site. Water temperature was gently increased from 11 to $16^{\circ} \mathrm{C}$ during the first 8 days of the experiment and then kept constant to $16^{\circ} \mathrm{C}$ afterwards. The limpets were fed daily with an average of $5.4 \mathrm{~L}$ of Isochrysis galbana [24.6 $\cdot 10^{9}$ cells per liter] mixed with $5.2 \mathrm{~L}$ of Chaetoceros gracilis [15.6 $\cdot 10^{9}$ cells per liter]. Half of this mixture was given at once during the day, after which water renewal was stopped for a few hours. The other half was distributed continuously during the night (slow automatic distribution). During week-ends ca. $10 \mathrm{~L}$ of the same microalgae mixture was distributed continuously. All plastic structures containing the stacks (main text Fig.1) were cleaned every 1-3 days.

Parentage analyses with software COLONY (Jones and Wang 2010)

Each larval release (represented by 48 larvae) was analyzed independently with the fulllikelihood approach with one run of "medium length" and "high likelihood precision". Since the experimental set-up (Fig. 1) allowed us to associate each larval release with a unique stack, all females of that particular stack were designed as candidate mothers in COLONY. The set of candidate fathers included all individuals of the stack but the most basal one (because any female but the most basal one in the stack could have produced male gametes prior to sex-change in the recent past and thus, through sperm storage, be the father of larvae produced during the experiment). We allowed both for polygyny and polyandry, setting the parentage prior (guess probability that the parent of an offspring is included in the candidate pool) to 1 for mothers. For fathers we assumed that ca.1-2 males outside a given stack could have contributed to reproduction 
(based upon results from Dupont et al. 2006; Le Cam et al. 2009), hence we set parentage prior to

$(I-1) /(I+2)$ where $I$ is the number of individuals in the focal stack. This choice of prior is

straightforward, but we note that this level of refinement for father parentage prior is inconsequential here because all mothers and a very large fraction of the fathers were genotyped. from the 33 stacks used in the breeding experiment (three individuals were disposed by error or lost before genotyping) and the 190 individuals that had been simultaneously sampled in the wild (that is, the second set of adults detailed in the main text). Random genotyping repetitions ( 20 to 98 per locus, data not shown) allowed us to detect a very high error rate for locus DAYN22, which was therefore removed from all analyses. With the remaining loci we set COLONY error settings to $1 \%$ for allelic dropouts and $0.1 \%$ for other genotyping errors (e.g. mutations or PCR-generated false alleles). polymorphic species such as many marine invertebrates (e.g.Hare et al. 1996; Lemer et al. 2011). Allowing for a small proportion of genotyping errors does not fully control for the effect of null alleles in COLONY analyses, because a single non-amplified allele in a parent is expected to be present in half of its progeny. However, such patterns are easily detected with parent/offspring data, especially with large family sizes. In our case the fact that the mother was necessarily in the pool of candidate parents greatly facilitated the detection of null alleles in the parents of the two sexes (see also Proestou et al. 2008).

Detailed description of the model 
closest to the females (individual number 3 in Fig. 5). We are interested in following the dynamics of reproductive success of this focal individual as a function of sex and time.

Time is divided into discrete units corresponding to the frequency of brood production by the females. Each female produces one brood per time unit, and we take for example one time unit $=$ one month. For simplicity the fecundity of the two females is equal and remains constant (depicting e.g. a situation where these two females are already quite large (and old) and do not grow anymore). Each female produces $N$ larvae at each time step. In all simulations we set $N=14000$ offspring (average fecundity from the empirical study).

Our focal individual will start its benthic life at time $t=0$ with shell length $L=0$ and obey a Von Bertalanffy growth increment of the form:

$$
L_{t+1}=L_{t}+\left(L_{\infty}-L_{t}\right)(1-\exp (-K))
$$

where parameters $L_{\infty}$ and $K$ were approximated from our growth data using Ford-Waldford method, that is, the linear regression of shell length taken at the onset of the experiment against shell length of all surviving individuals at day 251 (i.e. approximately 8 month). For simplicity and because there are no small females we used a unique growth model fitted with empirical data from both sexes, giving $L_{\infty} \approx 56.48$ and $K \approx 0.043$. Our focal individual will thus grow at each time step. Because we want to look at the effect of sperm storage alone (and not the complexities associated with other aspects of the mating system or stacking behavior), we considered that our focal individual is directly in contact with a female (Fig. 5). Hence size/age will have no influence on reproductive success until the individual turns female. After that, the fecundity of this individual will depend directly on its size (details below).

At each time step we calculate the reproductive success of our focal individual through its male function, and, if it has already changed sex, through both male and female functions (similarly to the measure of reproductive success defined with our empirical data). The timeframe of our 
model is arbitrarily set to 100 time steps (a period that is long enough to include the optimal timing of sex change in all simulations and is in the range of $C$. fornicata life expectancy).

\section{Reproductive success through the male function}

Before sex-change of our focal individual, the two males compete for fertilizing female broods. Each female has a sperm storage reserve that contains a proportion $p$ of sperm from our focal male. In a first step (referred to as model 1, Fig. 5) we assume that the two female sperm reserves follow the same dynamics. At each time step $t$, a fraction $m$ of sperm is lost through sperm mortality (that includes consumption for fertilization of the eggs) and replaced by sperm produced by each male in proportions $\varphi$ from our focal male and $1-\varphi$ from its competitor (Fig. 5). Within each female's sperm storage organ, the proportion $p$ of sperm from our focal male thus follows:

$$
p_{t+1}=p_{t}(1-m)+m \varphi
$$

This model depicts a situation where the sperm storage organ is elastic and new sperm accumulate as a random mixture, or storage is limited but sperm is replaced proportionally to male contributions (akin to a "fair raffle" type of sperm competition, Parker 1998, without sperm precedence).

At each time step $t$ the reproductive success of our focal male is:

$$
W_{t}^{\hat{0}}=2 p_{t} N
$$

that is, the combined fecundity of the two females weighted by the proportion that the sperm of the focal male represents in the sperm stores. With this definition, the reproductive success through male function depends only on male-male competition. It is independent of the size or age of the focal male (in particular there is no immature phase, we take the simplification that the focal male is fully mature at time $t=0$ ). The equilibrium state for the proportion of sperm from our focal male stored in each female is $p_{e q}=\varphi$ and the reproductive success of this male at equilibrium is $W_{e q}^{\hat{o}}=2 \varphi N$ 

in equation S2) and the dynamics of sperm reserves becomes:

$$
p_{t+1}=p_{t}(1-m)
$$

98

99

The fitness (eq. S3) of our focal individual (now female) through male function will thus decline at rate $m$ until it becomes null when all its sperm has been replaced by sperm from the other male.

Reproductive success through the female function

Contrary to male fitness, female's fecundity is constrained by its size (Li and Pechenik 2007; Proestou et al. 2008; Richard et al. 2006). Here we used the linear relationship reported by Li and Pechenik (2007) to define the reproductive success of our focal individual as a function of shell length when it has turned female: $W_{t}^{\dagger}=558.4 \times L_{t}-10380$. Negative values, predicted for $L_{t}<18.6 \mathrm{~mm}$, were set to 0 .

\section{Estimation of sperm mortality $m$ from empirical data}

In our models a proportion $m$ of sperm is lost from a female's sperm store each time that a new brood is produced. That sperm may (model 1 in Fig. 5) or may not (model 2) be replaced with new sperm. This parameter $m$ appears to be very influential for the effect of sperm storage on the timing of the sex-change. There is one situation where $m$ could be estimated from our parentage data. The female at the base of stack 1 repeatedly produced broods partly sired by the second individual of that stack, which was a female (see a cartoon of this stack in Fig. 8; we know that individual 2 was a female because it produced broods itself early in the experiment). Hence the father of these broods was already a female when the experiment began in January, but its sperm stored in the basal female (female 1) was repeatedly used to fertilize that female's eggs. For the reproductive success analyses presented in this study we already had parentage data for 4 consecutive broods produced 
by female 1 (with 16 to 19 days between successive broods, Fig. 8). We genotyped and analyzed one additional brood produced 180 days later by the same female. We estimated the proportion $p$ of larvae fathered by female 2 in each brood ( $n=16$ to 87 larvae genotyped per brood). The standard

121 error of each estimate is given by $s_{p}=\sqrt{\frac{p(1-p)}{n-1}}$.

122 We used these data to fit a decay model of the form $p_{t}=p_{0}(1-m)^{t}$ where $p_{0}$ is the fitted proportion of sperm from female 2 used by female 1 for the first brood analyzed (we arbitrarily set this first brood at time $t=0$, Fig. S8). This model gives a remarkable fit ( $\mathrm{p}$-value $=0.005$, adjusted $\mathrm{R}^{2}=$ 0.93 ) for $p_{0}=0.21$ and $m=0.14$. Note that the timing of the first brood (and hence $p_{0}$ ) doesn't have any importance. For instance we obtain $m=0.14$ also without considering the first brood ( $p$ value $=0.03$, adjusted $R^{2}=0.90$ ). We don't know the rules that drive the decay in the contribution of individual 2 (e.g. sperm mortality with random replacement or more complex mechanisms involving sperm precedence or postcopulatory competition) but it gives us a remarkable quantitative example of how sperm storage allows a male to continue fathering offspring after sex-change. 
133 Figure S1: We observed an average brood size of ca. 14000 larvae, of which only a minute fraction

134 could be genotyped for parentage assignment and reproductive success analyses. Assuming that one 135 observation of a larval release corresponds to one brood produced by a single female and that each 136 sampled larva can be genotyped and correctly assigned to its parents, how many larvae should be sampled to detect all the fathers that contributed to a given brood? The probability of detecting at least one offspring of a father that contributed a fraction $p$ of a brood can be calculated as 1 $(1-p)^{n}$ with sample size $n$. Panel A shows this probability for sample sizes 32,48 , and 96 larvae for theoretical sire contributions [0-0.25]. Panel B shows the expected number of offspring of a given father as a function of that father's contribution to a brood, within sample of size 32,48 , or 96 larvae.

Figure S2: Distribution of mating success (number of successful mating partners) of adult Crepidula fornicata (31 males and 37 females from 8 stacks). Females that reproduced only through the female function are pictured with white bars. Some females (black bars) have both male and female mating partners (or even female partners only), because before changing sex they produced male gametes that are still used by other females (sperm storage effect).

Figure S3: Distribution of the proportion of larvae sired by a given male in a given brood. 
Figure S5: Effect of stack structure on different components of reproductive success. A) Distribution of the distance between mating partners (that is, the number of other individuals that separate two mating partners in a stack). During the experiment we randomly observed 145 matings involving 101 individuals from 22 stacks. The distribution of distances for these observations (dark grey bars) can be compared to the expected distribution (light grey) under the null hypothesis of random mating between males and females within the same 22 stacks, taking into account the composition of each stack. B) Effect of the distance between parents of a given brood on the proportion of larvae sired by the father ( $n=125$ proportions involving 32 broods and 16 fathers). The two kinds of triangle symbols correspond to apparent outliers that were all due to only two males within a single stack showing a particularly strong curvature that may have facilitated internal fertilization between distant individuals.

Figure S6: Effect of sperm mortality $(m)$ on the gain in lifetime reproductive output $(\Delta \omega)$ for an individual that would change sex at some time $t$ (with sperm storage occurring), by comparison with an individual that follows the optimal strategy in the absence of sperm storage. These results were obtained under the conditions of model 1 (see main text and Figure 5), that is, where the sperm of the focal male that is stored in females is progressively replaced by sperm from another donor.

Figure S7: Effect of sperm mortality $(m)$ on the gain in lifetime reproductive output $(\Delta \omega)$ for an individual that would change sex at some time $t$ (with sperm storage occurring), by comparison with an individual that follows the optimal strategy in the absence of sperm storage. These results were obtained under the conditions of model 2 (see main text and Figure 5), that is, where only one female progressively replaces the sperm from the focal male by sperm from another donor, while the other female does not receive any new sperm. 
Figure S1

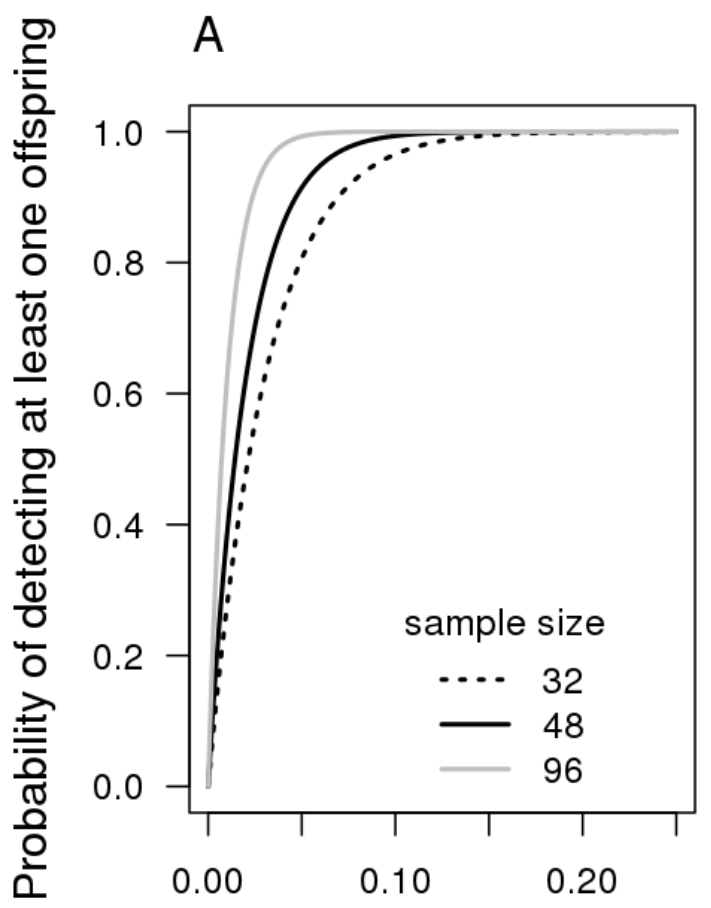

Actual father contribution

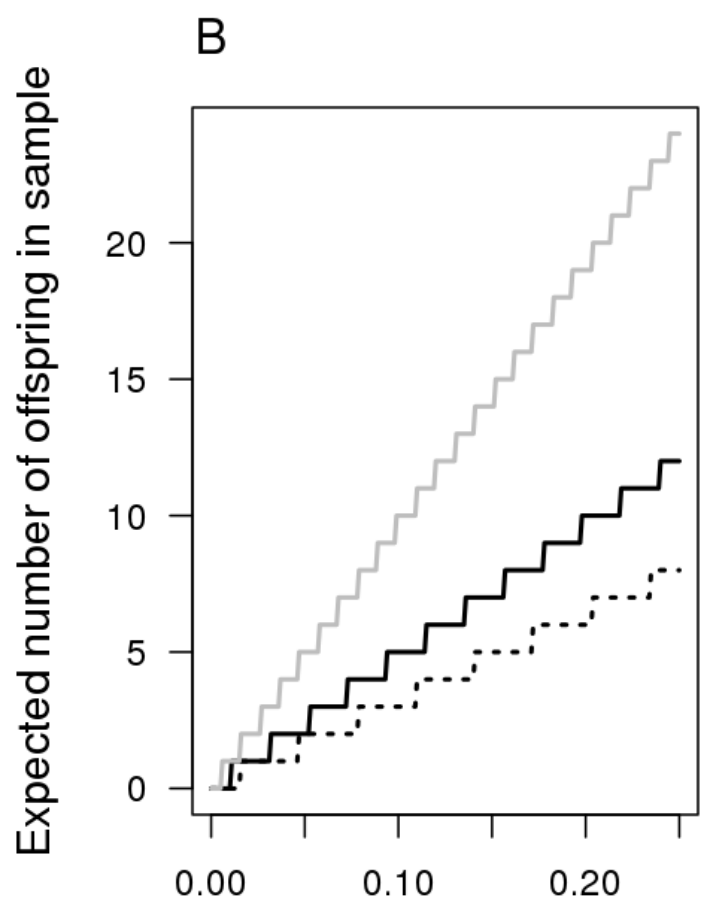

Actual father contribution

Figure S1: We observed an average brood size of ca. 14000 larvae, of which only a minute fraction could be genotyped for parentage assignment and reproductive success analyses. Assuming that one observation of a larval release corresponds to one brood produced by a single female and that each sampled larva can be genotyped and correctly assigned to its parents, how many larvae should be sampled to detect all the fathers that contributed to a given brood? The probability of detecting at least one offspring of a father that contributed a fraction $p$ of a brood can be calculated as $1-$ $(1-p)^{n}$ with sample size $n$. Panel A shows this probability for sample sizes 32, 48, and 96 larvae for theoretical sire contributions [0-0.25]. Panel B shows the expected number of offspring of a given father as a function of that father's contribution to a brood, within sample of size 32, 48, or 96 larvae. 
Figure S2

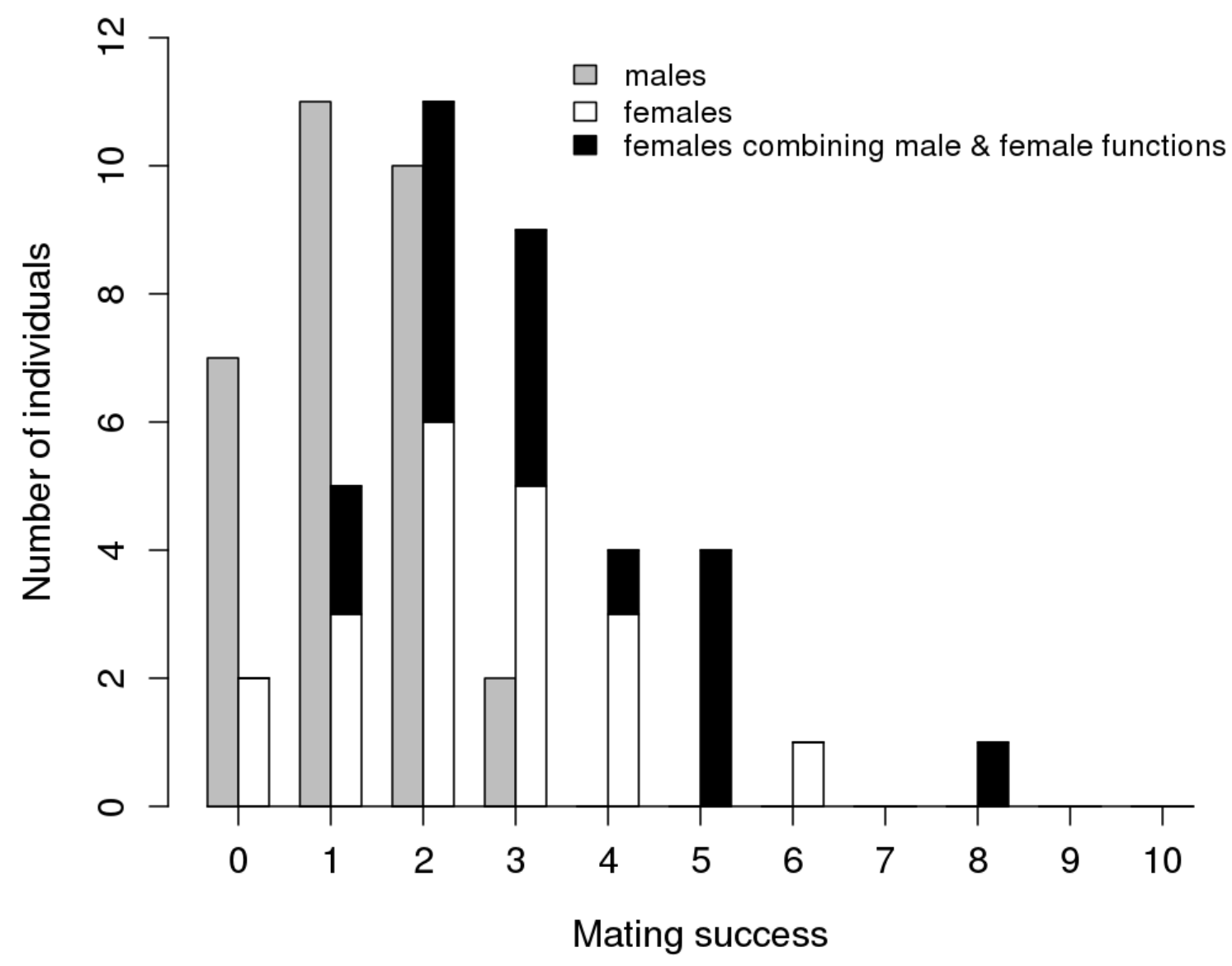

Figure S2: Distribution of mating success (number of successful mating partners) of adult Crepidula fornicata (31 males and 37 females from 8 stacks). Females that reproduced only through the female function are pictured with white bars. Some females (black bars) have both male and female mating partners (or even female partners only), because before changing sex they produced male gametes that are still used by other females (sperm storage effect). 
Figure S3

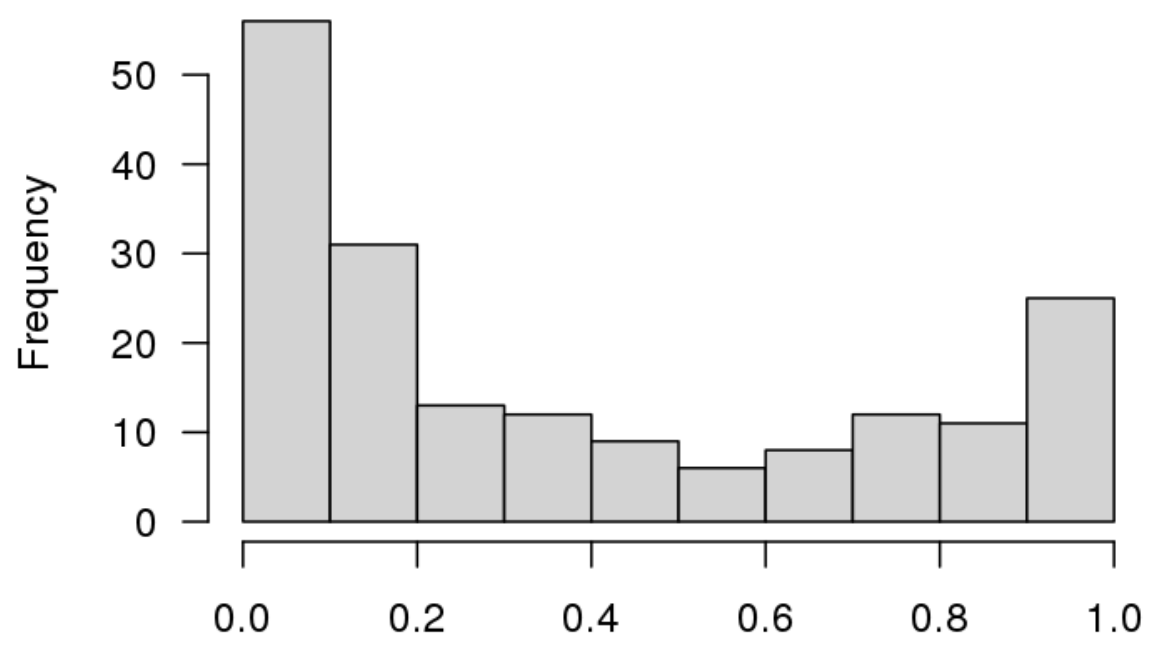

Proportion of larvae sired by a male within broods

Figure S3: Distribution of the proportion of larvae sired by a given male in a given brood. 
Figure S4

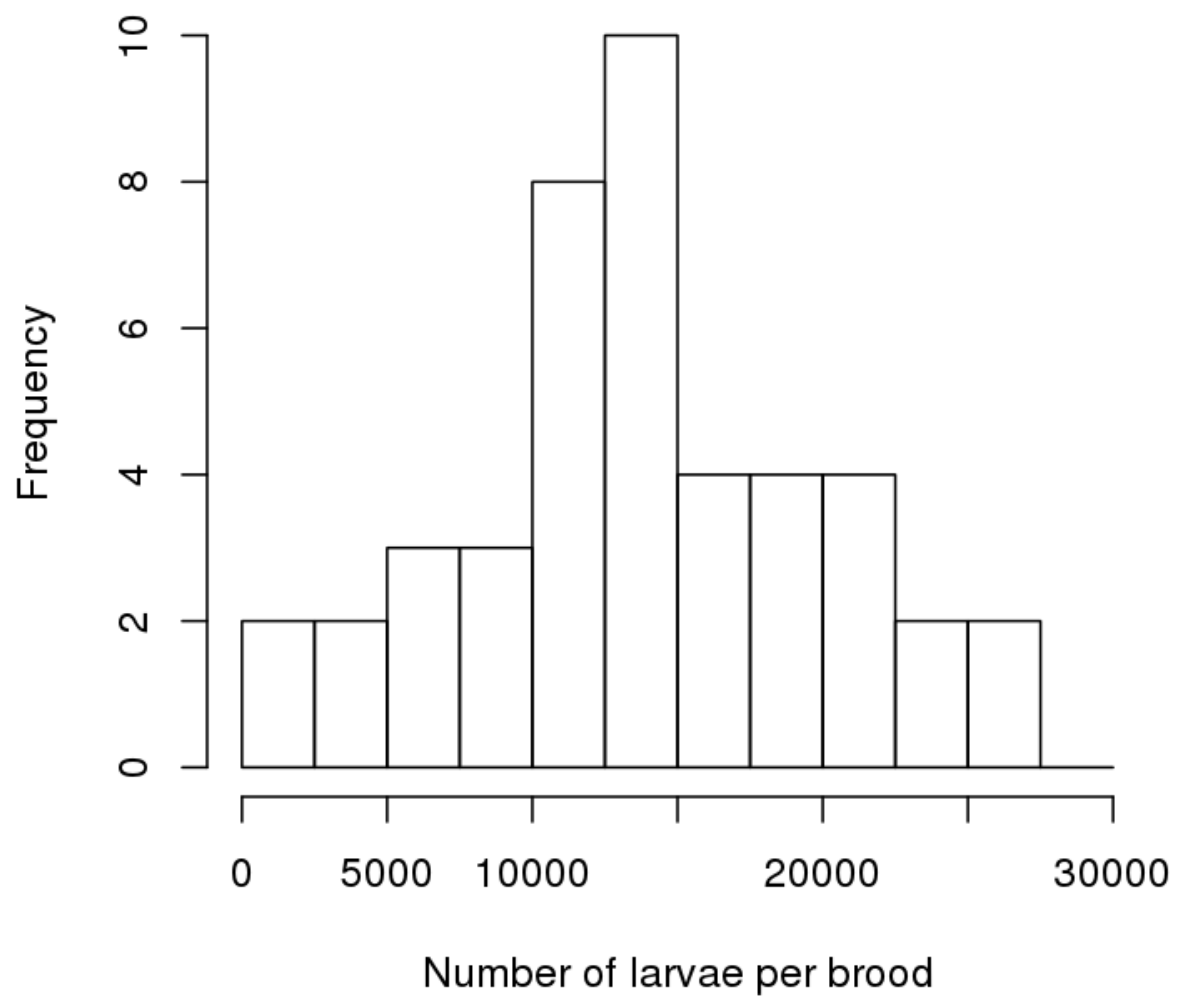

Figure S4: Distribution of brood size ( $n=44$ broods produced by 24 females from 8 stacks). 

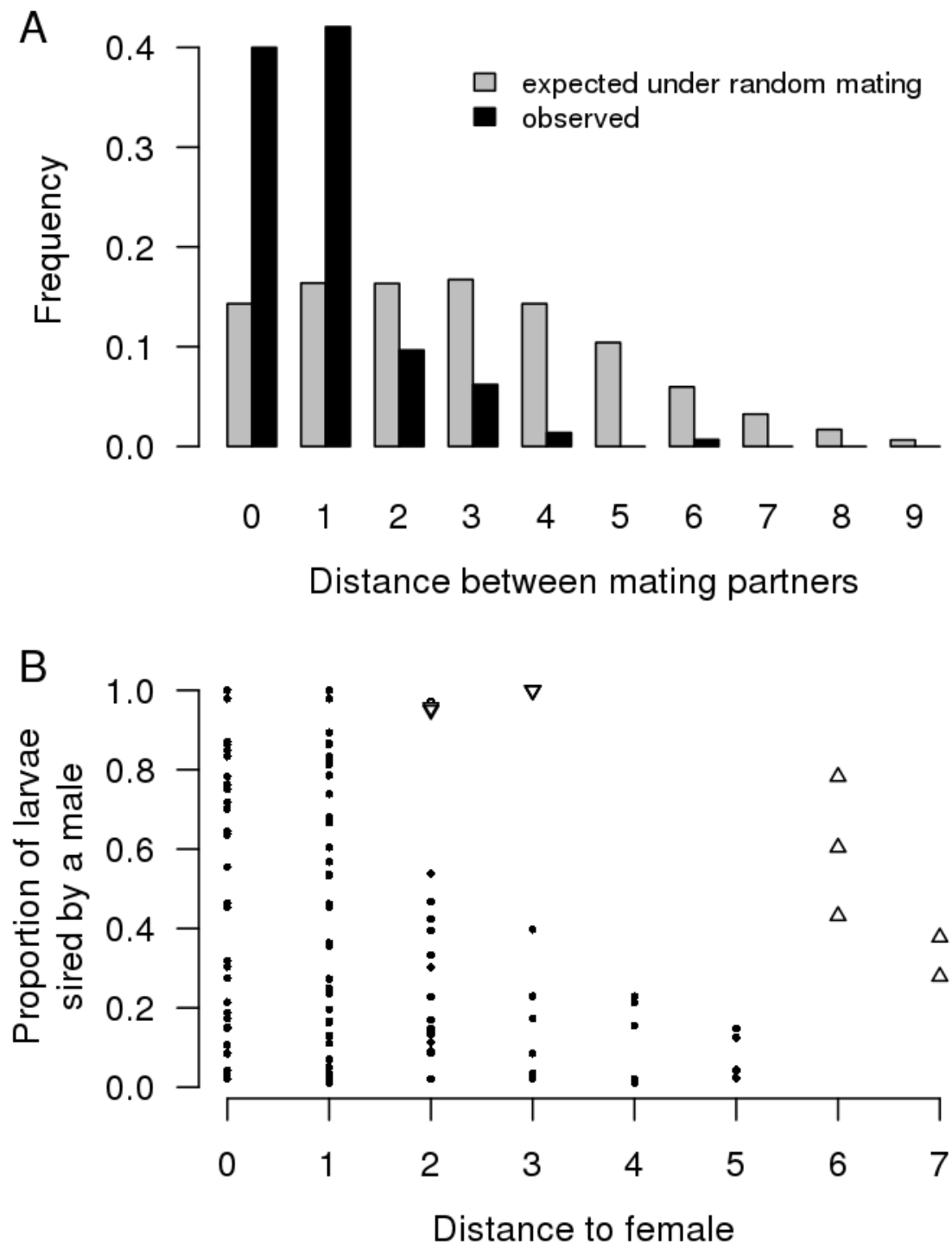

Figure S5: Effect of stack structure on different components of reproductive success. A) Distribution of the distance between mating partners (that is, the number of other individuals that separate two mating partners in a stack). During the experiment we randomly observed 145 matings involving 101 individuals from 22 stacks. The distribution of distances for these observations (dark grey bars) can be compared to the expected distribution (light grey) under the null hypothesis of random mating between males and females within the same 22 stacks, taking into account the composition of each stack. B) Effect of the distance between parents of a given brood on the proportion of larvae sired by the father ( $n=125$ proportions involving 32 broods and 16 fathers). The two kinds of triangle symbols 
correspond to apparent outliers that were all due to only two males within a single stack showing a particularly strong curvature that may have facilitated internal fertilization between distant individuals. 
Figure S6

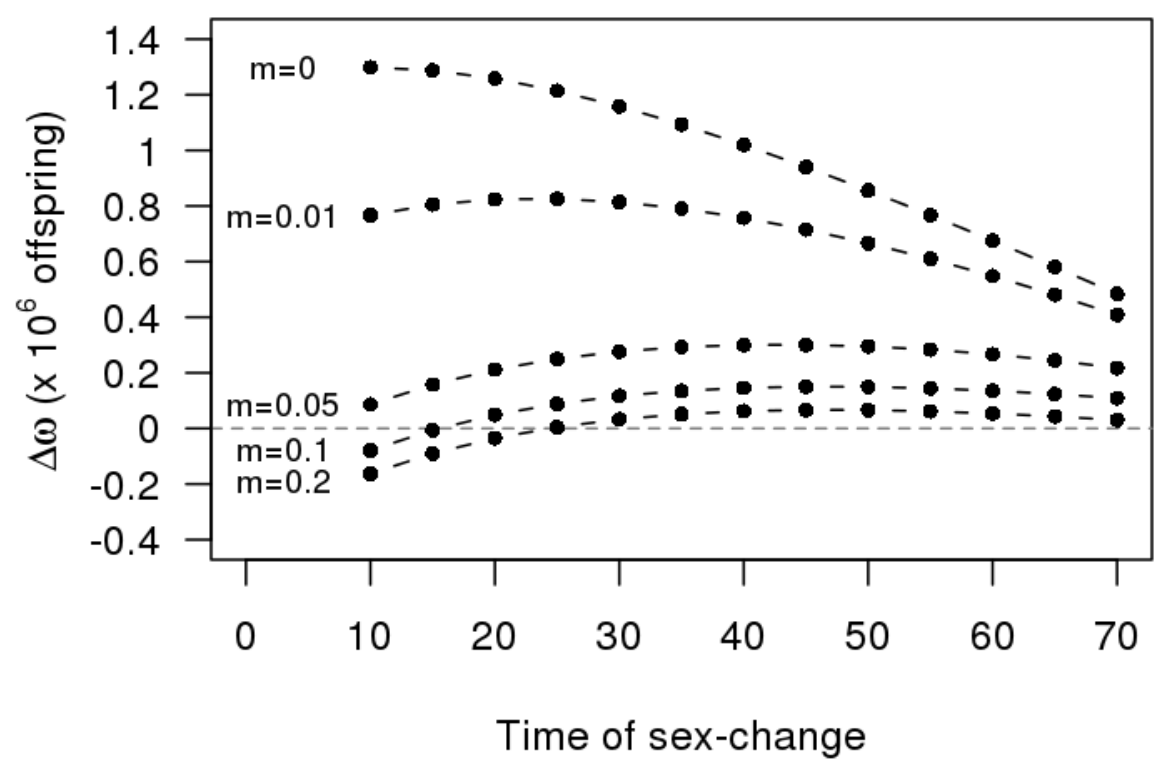

Figure S6: Effect of sperm mortality $(m)$ on the gain in lifetime reproductive output $(\Delta \omega)$ for an individual that would change sex at some time $t$ (with sperm storage occurring), by comparison with an individual that follows the optimal strategy in the absence of sperm storage. These results were obtained under the conditions of model 1 (see main text and Figure 5), that is, where the sperm of the focal male that is stored in females is progressively replaced by sperm from another donor. 
Figure S7

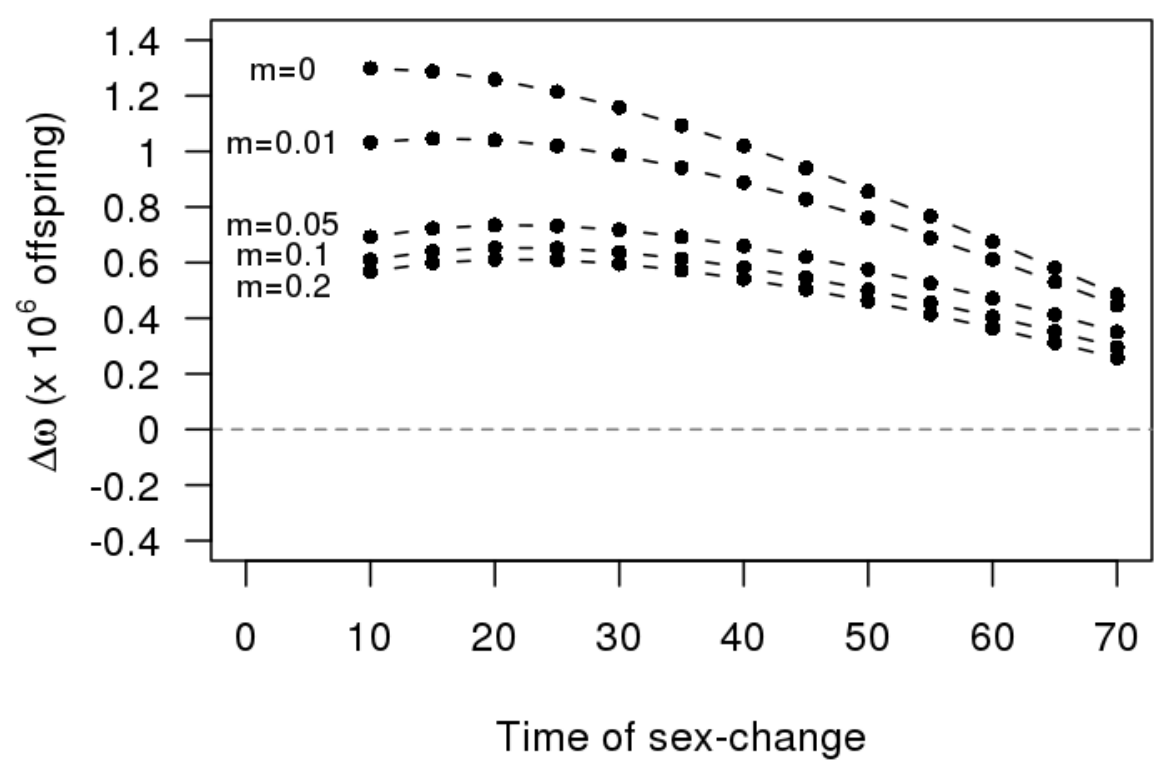

Figure S7: Effect of sperm mortality $(m)$ on the gain in lifetime reproductive output $(\Delta \omega)$ for an individual that would change sex at some time $t$ (with sperm storage occurring), by comparison with an individual that follows the optimal strategy in the absence of sperm storage. These results were obtained under the conditions of model 2 (see main text and Figure 5), that is, where only one female progressively replaces the sperm from the focal male by sperm from another donor, while the other female does not receive any new sperm. 


\section{References for supplementary material}

Dupont, L., J. Richard, Y.-M. Paulet, G. Thouzeau, and F. Viard. 2006. Gregariousness and protandry promote reproductive insurance in the invasive gastropod Crepidula fornicata: evidence from assignment of larval paternity. Molecular Ecology 15:3009-3021.

Hare, M. P., S. A. Karl, and J. C. Avise. 1996. Anonymous nuclear DNA markers in the American oyster and their implications for the heterozygote deficiency phenomenon in marine bivalves. Molecular Biology and Evolution 13:334-345.

Jones, O. R., and J. L. Wang. 2010. COLONY: a program for parentage and sibship inference from multilocus genotype data. Molecular Ecology Resources 10:551-555.

Le Cam, S., J. Pechenik, M. Cagnon, and F. Viard. 2009. Fast vs. slow larval growth in an invasive marine mollusc: does paternity matter? Journal of Heredity 100:455-464.

Lemer, S., E. Rochel, and S. Planes. 2011. Correction Method for Null Alleles in Species with Variable Microsatellite Flanking Regions, A Case Study of the Black-Lipped Pearl Oyster Pinctada margaritifera. Journal of Heredity 102:243-246.

Li, W., and J. A. Pechenik. 2007. Effect of inbreeding on reproduction and juvenile performance in two marine gastropods with contrasting reproductive patterns. Marine Ecology Progress Series 346:219-234.

Parker, G. A. 1998. Sperm competition and the evolution of ejaculates: towards a theory base, Pages 3-54 in T. R. Birkhead, and A. P. Moller, eds. Sperm competition and sexual selection. San Diego, Academic Press.

Proestou, D. A., M. R. Goldsmith, and S. Twombly. 2008. Patterns of male reproductive success in Crepidula fornicata provide new insight for sex allocation and optimal sex change. Biological Bulletin 214:192-200.

Richard, J., M. Huet, G. Thouzeau, and Y. M. Paulet. 2006. Reproduction of the invasive slipper limpet, Crepidula fornicata, in the Bay of Brest, France. Marine Biology 149:789-801. 
